Article

\title{
Implementation of Dynamic Virtual Inertia Control of Supercapacitors for Multi-Area PV-Based Microgrid Clusters
}

\author{
Li Yang and Zhijian $\mathrm{Hu}$ * \\ School of Electrical Engineering and Automation, Wuhan University, Wuhan 430072, China; \\ yangli_1003@outlook.com \\ * Correspondence: zhijian_hu@163.com
}

Received: 8 March 2020; Accepted: 6 April 2020; Published: 18 April 2020

check for updates

\begin{abstract}
In order to improve the dynamic stability of multi-area microgrid (MG) clusters in the autonomous mode, this study proposes a novel fuzzy-based dynamic inertia control strategy for supercapacitors in multi-area autonomous MG clusters. By virtue of the integral manifold theory, the interactive influence of inertia on dynamic stability for multi-area MG clusters is explored in detail. The energy function of multi-area MG clusters is constructed to further analyze the inertia constant. Based on the analysis of the mechanism, a control strategy for the fuzzy-based dynamic inertia control of supercapacitors for multi-area MG clusters is further proposed. For each sub-microgrid (sub-MG), the gain of the fuzzy-based dynamic inertia control is self-tuned dynamically, with system events being triggered, so as to flexibly and robustly enhance the dynamic performance of the multi-area MG clusters in the autonomous mode. To verify the effectiveness of the proposed control scheme, a three-area photovoltaic (PV)-based MG cluster is designed and simulated on the MATLAB/Simulink platform. Moreover, a comparison between the dynamic fuzzy-based inertial control method and an additional droop control method is finally presented to validate the advantages of the fuzzy-based dynamic inertial control approach.
\end{abstract}

Keywords: microgrid (MG) clusters; virtual inertia; supercapacitors; dynamic stability; fuzzy control

\section{Introduction}

Microgrids (MGs) consist of distributed generators (DGs), energy storage systems (ESSs) and loads, and they can operate in both grid-connected and autonomous modes [1]. However, a single MG can only accommodate a limited capacity of distributed renewable generation, e.g., photovoltaic (PV). To solve this issue, the concept of MG clusters has recently been proposed. In [2], the concept of MG clusters was first proposed and investigated, and it was concluded that they can provide the benefits of lower costs and emissions. With operation in autonomous mode, MG clusters can receive power support from other sub-microgrids (sub-MGs) in order to maintain the power balance. For example, two neighboring sub-MGs, operating in autonomous mode, can exchange power with each other in the case of an emergency situation, as in [3]. However, because most control strategies for renewable generation cannot respond to the frequency deviation of power systems, the physical inertia of MG clusters is obviously lower than that of the traditional synchronous generator-based power system. As for the MG clusters, the absence of natural rotational inertia in power electronic interfaces may result in the issue of dynamic stability when the autonomous mode is adopted [4-6].

The capacity and inertia of MG clusters are relatively low [7]. Any interruptions (e.g., load variations and short-circuit faults) would adversely affect the frequency stability of MG clusters in the autonomous mode. Therefore, it is necessary for MG clusters to increase their system inertia and 
enhance their frequency stability against disturbances. Virtual inertia has been widely proposed in the literature as a solution for low inertia issues. The concept of the virtual synchronous generator (VSG) is applied to emulate synchronous generator characteristics in [8,9]. In [10], VSG is shown to be a special case of droop control, which mimics the rotor inertia and damping effect of synchronous generators. VSGs are flexible in choosing parameters such as inertia, the damping coefficient, reactance and various time constants [11,12], but the setting of control parameters is complex. Additionally, droop controllers used in the operation of distributed energy resources (DERs) have also been shown to provide virtual inertia under certain conditions [13]. Furthermore, other inverter-interfaced generators, such as PV generation, can also supply synthetic inertia through working below the maximum power point (MPP) [14]. The required energy is obtained through the curtailed operation of PV, but the operation of working below MPP may not be cost-efficient [15]. Thus, considering the maximum utilization of DGs and the capacity of the inverter, installing an ESS is a helpful and significant way to improve the dynamic performance of MG clusters [16].

As the backup power reserve equipment, energy storage systems are important to enhance the flexibility of MG cluster operation, and they can be controlled to quickly charge or discharge power. Hence, an ESS (e.g., batteries or supercapacitors) is able to effectively offer frequency support in MG clusters and smooth the power fluctuations caused by the intermittent and stochastic output power of renewable generations $[17,18]$. As a controlled source, ESSs are used for keeping constant voltage and frequency control (V/f) in MGs. Furthermore, an advantage of installing an ESS has been verified, which is the improvement of the frequency and voltage responses compared with those in the case of no ESS [19]. However, the application of ESSs imposes additional challenges for MG clusters, such as the need for a strategy that properly maintains the state of charge (SOC) of ESSs within a secure operation. In [20], active power curtailment on both the generation and demand sides is applied to avoid the overcharge and discharge of ESSs. Therefore, in order to guarantee the safe operation of ESSs, it is important to exploit the effective attributes of ESSs that can provide the same frequency support as synchronous generators.

Moreover, small-signal stability is always a dynamic stability issue for interconnected power systems. Due to the complex interconnection among sub-MGs, MG clusters could also suffer the risk of poorly-damped oscillation. For example, the small-signal stability issue can be triggered by the poor coordination of multiple frequency controllers and power sharing among DGs, which consequently leads to undamped frequency oscillations in the span of a few seconds to a few minutes [21]. References [22,23] demonstrate that a large droop coefficient is beneficial to speed up the power response of DGs and improve the dynamic performance of the whole system, but it could reduce the damping ratio of low-frequency oscillation modes in some cases. Aiming to avoid such periodic instability of MG clusters, there exists a great desire to investigate an effective control scheme for alleviating the small-signal stability issue.

Based on the aforementioned discussion, in the autonomous operation of MG clusters, the absence of natural rotational inertia and the complex interconnection among sub-MGs may lead to the issue of dynamic stability. To achieve an improvement in dynamic stability, the purpose of this study is to tackle three significant problems for MG cluster dynamic stability: (i) how the low inertia of MG clusters affects the dynamic stability in the autonomous mode, (ii) how to use the virtual inertia of supercapacitors to address the frequency stability problem caused by low inertia, and (iii) how to obtain a sufficient damping performance in the MG cluster by adjusting its inertia constant. The main contributions of this study are summarized as follows:

1. The definition of virtual inertia provided by ESSs, such as supercapacitors, in sub-MGs is analyzed in detail, and the relationship between the SOC, the frequency of AC MG clusters and the power of ESSs is established. This analysis provides the theoretical basis for virtual inertia control and indicates that virtual inertia control for ESSs significantly enhances the frequency stability of MG clusters.

2. For multiple sub-MGs in an MG cluster in autonomous mode, the influence of inertia on small-signal stability is analyzed by the integral manifold theory. This detailed analysis describes 
the dynamic process of the influence of inertia on small-signal stability, and it provides the theoretical inspiration for the virtual inertia dynamic control method.

3. The energy function of multi-area MG clusters is constructed to further analyze the inertia constant. Based on the theoretical analysis, fuzzy-based dynamic inertial control is proposed to regulate the inertia constant dynamically with variable events, so as to improve the dynamic stability for multiple sub-MGs in an MG cluster in autonomous mode. Analysis of the dynamic inertia constant can provide fundamental theoretical support to analyze the dynamic stability of multi-area MG clusters. The proposed fuzzy-based dynamic inertial control strategy is an extension of previous study, and it is more effective than the traditional droop control.

The rest of this paper is organized as follows: Section 2 describes the architectures of MG clusters. Next, Section 3 discusses the mechanism of the influence of inertia on dynamic stability. Furthermore, a fuzzy-based dynamic inertia control strategy is proposed in Section 4. In Section 5, simulation results are presented to verify the effectiveness and advantages of the proposed control method. Finally, conclusions are drawn in Section 6.

\section{Architecture of MG Clusters}

Generally, three types of architecture have been proposed for MG clusters, as shown in Figure 1 [7]:

(1) Parallel Connected MGs with an external grid (PCM): all sub-MGs are connected to the same external grid, where each MG has only one point of common coupling (PCC), as depicted in Figure 1a. In the grid-connected mode, the external grid is a strong aid for these sub-MGs to guarantee their stability against potential emergency disturbances. However, when the autonomous mode is adopted, each isolated sub-MG would suffer the risk of security stability as it loses the only external power support.

(2) Grid Series Interconnected MGs (GSIM): similar to the topology of traditional power grids, all sub-MGs are interconnected through tie-lines and based on the point to point structure. In Figure 1b, each sub-MG can get power support from other sub-MGs. Therefore, as for the autonomous mode, GSIM could have a better dynamic performance in comparison with that of the architecture of PCM.

(3) Mixed Parallel-Series Connection (MPSC): as observed in Figure 1c, the MPSC is the combination of the PCM and GSIM. Because the external grid and interconnected sub-MGs can simultaneously provide the necessary power support, MPSC is currently a reliable and promising architecture for MG clusters.

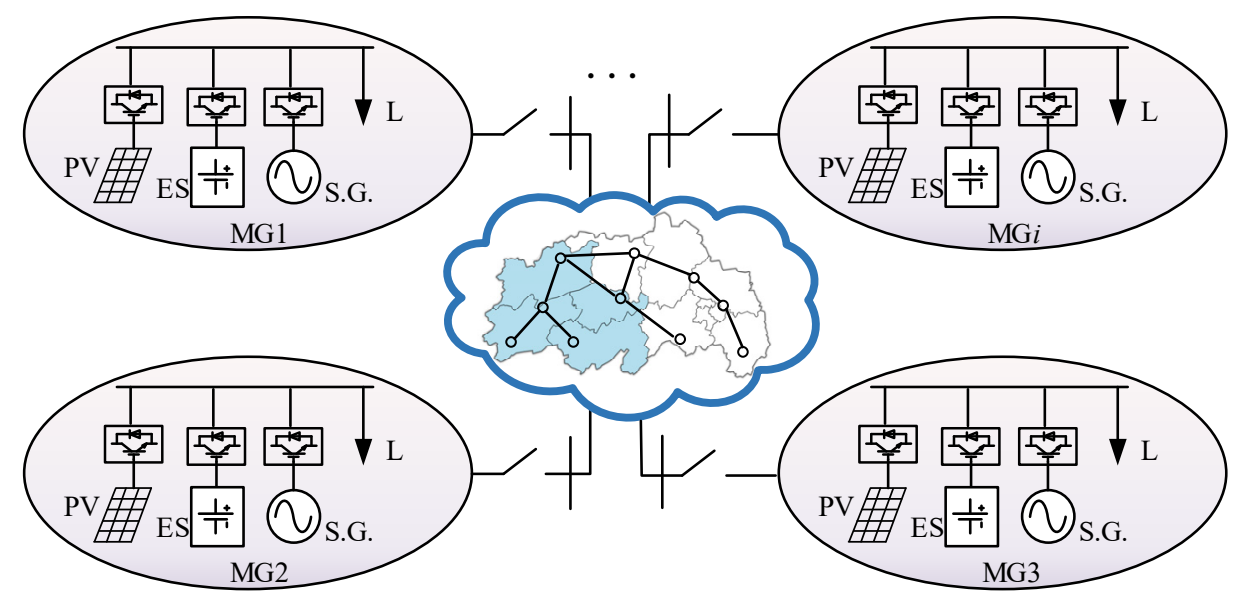

(a)

Figure 1. Cont. 


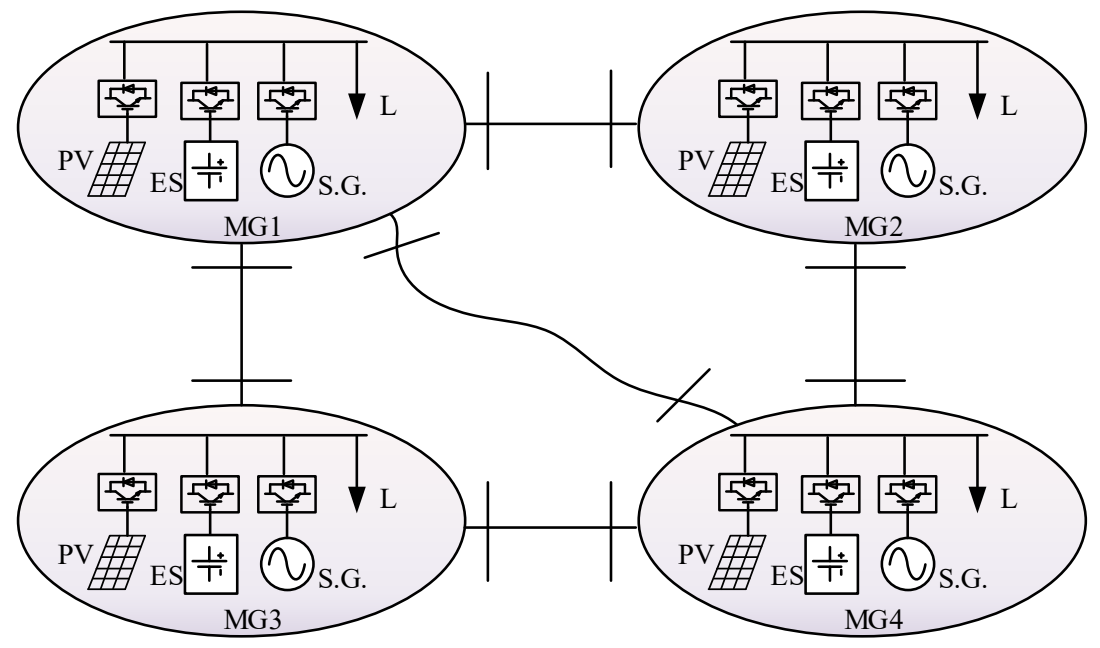

(b)

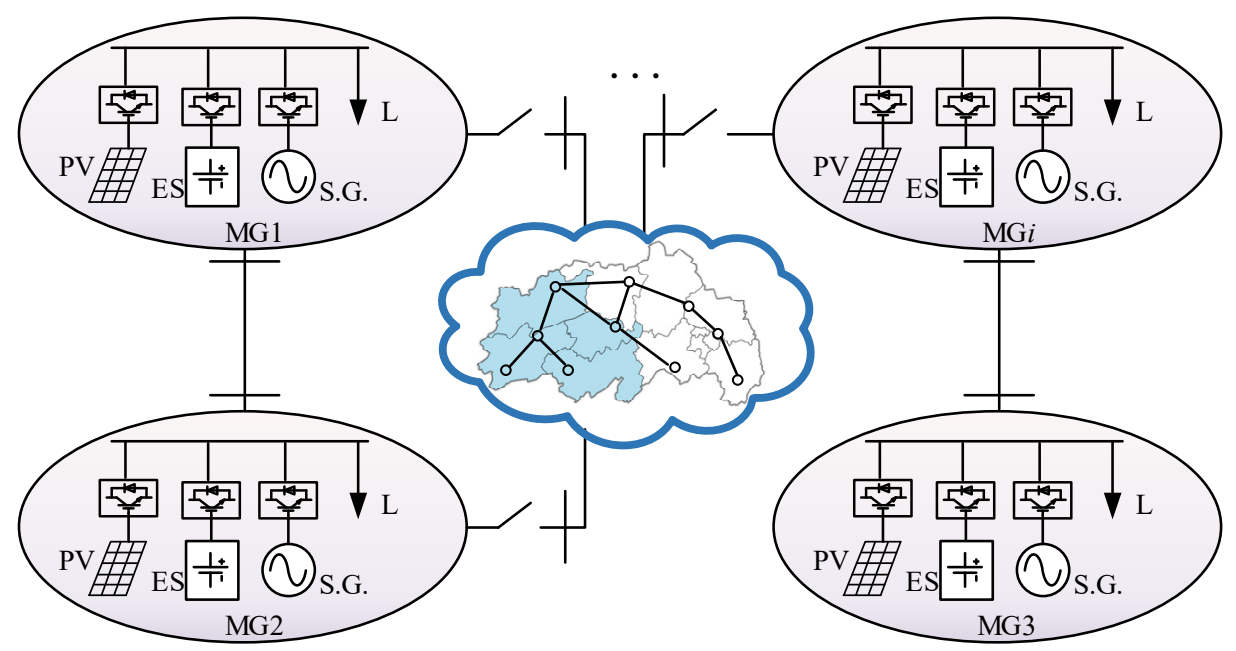

(c)

Figure 1. The structure of microgrid (MG) clusters: (a) parallel connected MGs, (b) grid series interconnected MGs, and (c) the mixed parallel-series connection.

In this study, the dynamic stability of MPSC-based MG clusters is mainly investigated. When sub-MGs are interconnected to the external grid (namely grid-connected mode), MPSC-based MG clusters can get sufficient power support through PCCs and guarantee the satisfactory dynamic performance of the whole system. If isolating switches are open, the operation mode of MPSC-based MG clusters turns into the autonomous mode. In this case, each sub-MG can be termed as an equivalent synchronous generator of traditional power grids. However, due to the low inertia of sub-MGs, it is difficult for MPSC-based MG clusters to prevent the fast collapse of frequency. On the other hand, the interconnecting sub-MGs may lead to the poorly-damped oscillation of tie-line power, which will further deteriorate the stability of MG clusters. Hence, the dynamic stability of MG clusters should be paid more attention.

Therefore, the dynamic stability of MPSC-based MG clusters becomes the issue of concern in this study, of which the mechanism and control strategy will be addressed below.

\section{Dynamic Stability Analysis of PV-Based MG Cluster}

\subsection{Inertia of Three-Area PV-Based MG Clusters}

The MPSC-based MG cluster is used as a case study in this work, the layout of which is shown in Figure 2. Each sub-MG consists of PV generations, a supercapacitor, a microturbine (synchronous 
generators) and loads. All PV generations operate in MPPT mode and transfer power through the $\mathrm{DC} / \mathrm{DC}$ converter and DC/AC converter, which means there is no power reserve in the PV generations. In the autonomous mode, the isolating switch of the sub-MGs is open. However, due to the high penetration of DGs, the low inertia of the MG cluster becomes the main concern. To address this issue, it is significant to enhance the inertia of the MG clusters via appropriate controls.

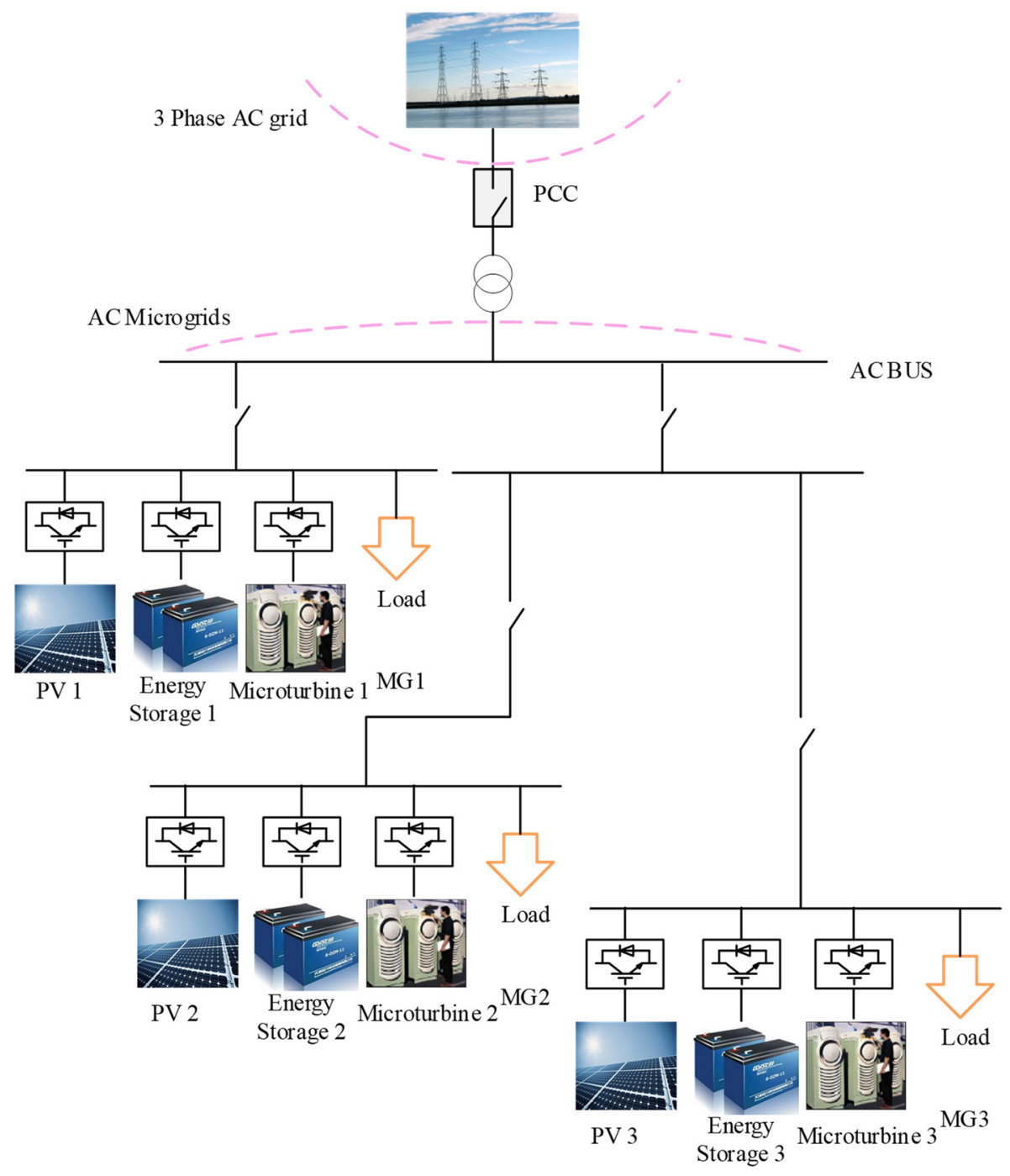

Figure 2. The structure of three-area photovoltaic (PV)-based MG Clusters.

The inertia constant of a power system is the ratio of rotor energy storage $E_{k \Sigma}$ to the total rated capacity of generators $S_{n \Sigma}$ under the synchronous angular speed, defined as:

$$
H_{\mathrm{S}}=\frac{E_{k \Sigma}}{S_{n \Sigma}}=\frac{\sum_{i=1}^{n}\left(J_{\mathrm{s}, i} \omega_{\mathrm{e}}^{2} / 2 p_{\mathrm{n}, i}^{2}\right)}{S_{n \Sigma}}
$$

where $n$ is the number of synchronous generators in the power system, $J_{\mathrm{s}, i}$ and $p_{\mathrm{n}, i}$ are the generator $i$ moment of inertia and the number of pole-pairs respectively, $E_{k \Sigma}$ is the rotational kinetic energy of all synchronous generators, and $S_{n \Sigma}$ is the total nominal generation capacity. It can be seen from Equation (1) that the inertia of MG clusters $H_{s}$ depends on the rotational kinetic energy synchronous generators' rotational kinetic energy $E_{k \Sigma}$.

Nevertheless, as a type of static generation unit, PV generations have no rotational mass to store kinetic energy. The higher penetration of PV generations in MG clusters leads to the replacement of 
traditional generators, which will significantly reduce inertia. Consequently, frequency stability in MG clusters is weakened with less inertia. On the other hand, although supercapacitors are also static generation units, they can keep a number of energy reserves and quickly regulate their output power. Therefore, the control of the supercapacitor naturally becomes an attractive and reasonable choice for effectively increasing the inertia of MG clusters.

According to [24], the supercapacitor is able to respond to the frequency changes in MG clusters and emulate the rotational inertia $J_{\mathrm{s}, i}$ of conventional synchronous generators. The energy $W_{c}$ of the supercapacitor and the equivalent virtual moment of inertia of the supercapacitor $J_{\text {vir_c }}$ are given as:

$$
\begin{gathered}
W_{\mathrm{c}}=\int u_{\mathrm{c}} C \frac{\mathrm{d} u_{\mathrm{c}}}{\mathrm{d} t} \mathrm{~d} t=\int \frac{u_{\mathrm{c}} C \mathrm{~d} u_{\mathrm{c}}}{\frac{\omega_{\mathrm{e}}}{p_{\mathrm{n}}^{2}} \omega_{\mathrm{e}}} \cdot \frac{\omega_{\mathrm{e}} \mathrm{d} \omega_{\mathrm{e}}}{p_{\mathrm{n}}^{2} \mathrm{~d} t} \mathrm{~d} t \\
=\int J_{\text {vir_c } \_} \mathrm{c} p_{\mathrm{e}}^{2} \mathrm{~d} \omega_{\mathrm{e}} \\
J_{\text {vir_c }}=\frac{u_{\mathrm{c}} C p_{\mathrm{n}}^{2} \mathrm{~d} u_{\mathrm{c}}}{\omega_{\mathrm{e}} \mathrm{d} \omega_{\mathrm{e}}}
\end{gathered}
$$

where $C$ is the capacitance of the supercapacitor, $\omega_{\mathrm{e}}$ is the synchronous angular speed of the equivalent generator, and $p_{\mathrm{n}}$ is the number of pole-pairs. Compared to Equation (1), it can be seen from Equations (2) and (3) that both synchronous generators and supercapacitors can release energy reserves $\left(E_{k \Sigma}\right.$ and $\left.W_{c}\right)$ against the frequency deviation. Hence, the value of the supercapacitor is equivalent to the kinetic energy of the equivalent synchronous generator set with $J_{\text {vir_c }}$ of inertia and $p_{\mathrm{n}}$ of pole-pairs.

Moreover, the virtual moment of inertia $J_{\text {vir_c }_{c}}$ can be calculated by the state of charge (SOC) of the supercapacitor $\rho_{s o c}$, as follows:

$$
\begin{gathered}
J_{v i r_{-} c}=\frac{u_{c} C p_{n}^{2} d u_{c}}{\omega_{e} d \omega_{e}}=\frac{u_{c N}^{2} C p_{n}^{2} d \rho_{s o c}}{2 \omega_{e} d \omega_{e}} \\
=\frac{\Delta \rho_{s o c}}{\Delta \omega_{e}} \cdot \frac{E_{c N} \omega_{e}}{2 E_{k}} J_{S}=k \cdot \frac{E_{c N} \omega_{e}}{2 E_{k}} J_{S} \\
k=\frac{\Delta \rho_{s o c}}{\Delta \omega_{e}}
\end{gathered}
$$

where $E_{c N}$ is the rated capacity of the supercapacitor, and $\omega_{e}$ is the synchronous angular speed of the equivalent generator. The virtual inertia coefficient $k$ can be adjusted by controlling the SOC of the supercapacitor. Then, the MG cluster can obtain short-time inertia support with a suitable charge/discharge of the supercapacitor.

Thus, considering the virtual inertia provided by supercapacitors, the total inertia constant $H_{t}$ of the system can be represented as:

$$
H_{t}=\left[H_{S} S_{N}+H_{\text {vir_c }} E_{c N}\right] / S_{t}
$$

where $H_{\text {vir_c }}$ is inertia constant of the supercapacitor, and $S_{t}$ is the nominal capacity of the MG clusters. It is evident from Equation (6) that the low inertia of MG clusters can be improved by introducing the virtual inertia of supercapacitors.

\subsection{Dynamic Stability Analysis of Three-Area PV-Based MG Clusters}

In the autonomous mode, there exists interconnection among the sub-MGs in the MPSC-based MG cluster. The small-signal stability of MG clusters is another issue, which can be observed by the tie-line power oscillations among the sub-MGs. Therefore, it is important to exploit the influence of inertia on small-signal stability and investigate how the inertia dynamically affects small-signal stability.

Based on the theory of small-signal analysis [1], the mechanism of how the inertia of sub-MGs affects the small-signal stability of MG clusters is discussed below. For the convenience of analysis, firstly, each sub-MG can be considered to be equivalent to a virtual synchronous generator $\left(\mathrm{VG}_{1}, \mathrm{VG}_{2}\right.$ and $\mathrm{VG}_{3}$, shown in Figure 3). Assuming that the $q$-axis transient electromotive force $E^{\prime}$ and mechanical power $P_{\mathrm{m} k}$ remain constant throughout the transient process, the rotor equations of $\mathrm{VG}_{1}, \mathrm{VG}_{2}$ and $\mathrm{VG}_{3}$ can be given by 


$$
\begin{aligned}
& \left\{\begin{array}{c}
2 H_{1} \frac{d \omega_{1}}{d t}=P_{m 1}-P_{e 1}-D_{1}\left(\omega_{1}-1\right) \\
\frac{d \delta_{1}}{d t}=\left(\omega_{1}-1\right) \omega_{0}
\end{array}\right. \\
& \left\{\begin{array}{c}
2 H_{2} \frac{d \omega_{2}}{d t}=P_{m 2}-P_{e 2}-D_{2}\left(\omega_{2}-1\right) \\
\frac{d \delta_{2}}{d t}=\left(\omega_{2}-1\right) \omega_{0}
\end{array}\right. \\
& \left\{\begin{array}{c}
2 H_{3} \frac{d \omega_{2}}{d t}=P_{m 3}-P_{e 3}-D_{3}\left(\omega_{3}-1\right) \\
\frac{d \delta_{3}}{d t}=\left(\omega_{3}-1\right) \omega_{0}
\end{array}\right.
\end{aligned}
$$

where $H_{1}, H_{2}$ and $H_{3}$ are the inertia constants; $P_{m 1}, P_{m 2}$ and $P_{m 3}$ are the mechanical powers; $P_{\mathrm{e} 1}, P_{\mathrm{e} 2}$ and $P_{\mathrm{e} 3}$ are the electromagnetic powers; $\omega_{1}, \omega_{2}$ and $\omega_{3}$ are the angular velocities; $\delta_{1}, \delta_{2}$ and $\delta_{3}$ are the power angles; $D_{1}, D_{2}$ and $D_{3}$ are the damping coefficients; and $\omega_{0}$ is the rated system angular velocity.

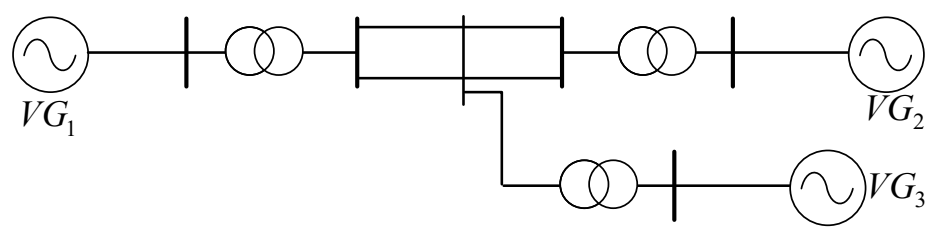

Figure 3. The equivalent circuit of three-area PV-based MG clusters.

According to the network power equation, the electromagnetic power equations of the equivalent three machine system can be expressed as

$$
\begin{aligned}
& P_{\mathrm{e} 1}=E_{1}^{2} G_{11}+E_{1} E_{2}\left(G_{12} \cos \delta_{12}+B_{12} \sin \delta_{12}\right)+E_{1} E_{3}\left(G_{13} \cos \delta_{13}+B_{13} \sin \delta_{13}\right) \\
& P_{\mathrm{e} 2}=E_{2}^{2} G_{22}+E_{1} E_{2}\left(G_{12} \cos \delta_{12}-B_{12} \sin \delta_{12}\right)+E_{2} E_{3}\left(G_{23} \cos \delta_{23}+B_{23} \sin \delta_{23}\right) \\
& P_{\mathrm{e} 3}=E_{3}^{2} G_{33}+E_{1} E_{3}\left(G_{13} \cos \delta_{13}-B_{13} \sin \delta_{13}\right)+E_{2} E_{3}\left(G_{23} \cos \delta_{23}-B_{23} \sin \delta_{23}\right)
\end{aligned}
$$

After linearizing Equations (7)-(9) around the equilibrium point of the MG clusters, the corresponding state-space equation of such a system is written as

$$
\begin{gathered}
{\left[\begin{array}{c}
\Delta \dot{\delta}_{13} \\
\Delta \dot{\delta}_{23} \\
\Delta \dot{\omega}_{1} \\
\Delta \dot{\omega}_{2} \\
\Delta \dot{\omega}_{3}
\end{array}\right]=\left[\begin{array}{ccccc}
0 & 0 & \omega_{0} & \omega_{0} & -\omega_{0} \\
0 & 0 & \omega_{0} & \omega_{0} & -\omega_{0} \\
-\frac{K_{11}}{2 H_{1}} & -\frac{K_{12}}{2 H_{1}} & -\frac{D_{1}}{2 H_{1}} & 0 & 0 \\
-\frac{K_{21}}{2 H_{2}} & -\frac{K_{22}}{2 H_{2}} & 0 & -\frac{D_{2}}{2 H_{2}} & 0 \\
-\frac{K_{31}}{2 H_{3}} & -\frac{K_{32}}{2 H_{3}} & 0 & 0 & -\frac{D_{3}}{2 H_{3}}
\end{array}\right]\left[\begin{array}{c}
\Delta \delta_{13} \\
\Delta \delta_{23} \\
\Delta \omega_{1} \\
\Delta \omega_{2} \\
\Delta \omega_{3}
\end{array}\right]} \\
K_{i j}=\frac{\partial P_{E i}}{\partial \delta_{j}}
\end{gathered}
$$

Equation (13) demonstrates that the rate of change of the state variables will be influenced by the inertia constants $H_{1}, H_{2}$ and $H_{3}$. Thus, further analysis is required to evaluate the influence of the adjustable inertial generation system on the frequency and power oscillation characteristics of the interconnected power grids.

In order to analyze the influence of inertia on the small-signal stability of the MG clusters, the integral manifold theory [25] is introduced in this paper. According to [26], the small-signal stability of interconnected power systems mainly depends on the poorly-damped oscillation mode, namely, the dominant oscillation mode. Therefore, the dominant mode among multiple sub-MGs is primarily investigated for MG clusters. Without undue loss of accuracy, a singular perturbation parameter $\varepsilon$ is defined according to Equation (15).

$$
\varepsilon=\frac{H_{3}}{H_{1}}
$$


Then, multiplying Equation (9) by $\varepsilon$, it is rewritten:

$$
\varepsilon \frac{d \Delta \omega_{3}}{d t}=\frac{-K_{31}}{2 H_{1}} \Delta \delta_{13}-\frac{K_{32}}{2 H_{1}} \Delta \delta_{23}-\frac{D_{3}}{2 H_{1}} \Delta \omega_{3}
$$

Supposing that the integral manifold is

$$
\Delta \omega_{3}=f\left(\Delta \delta_{13}, \Delta \delta_{23}, \Delta \omega_{1}, \Delta \omega_{2}, \varepsilon\right)
$$

and expressed as an exponential series in the neighborhood of $\varepsilon=0$, that is,

$$
\Delta \omega_{3}=f_{0}+\varepsilon f_{1}+\varepsilon^{2} f_{2}+\cdots
$$

the terms $f_{0}, f_{1}, \ldots$ of the series can be found, since the function $f$ must satisfy Equation (16), namely

$$
\begin{aligned}
& \left(\varepsilon \frac{\partial f_{0}}{\partial \Delta \delta_{13}}+\varepsilon^{2} \frac{\partial f_{1}}{\partial \Delta \delta_{13}}+\cdots\right) \frac{d \Delta \delta_{13}}{d t}+\left(\varepsilon \frac{\partial f_{0}}{\partial \Delta \delta_{23}}+\varepsilon^{2} \frac{\partial f_{1}}{\partial \Delta \delta_{23}}+\cdots\right) \frac{d \Delta \delta_{23}}{d t}+ \\
& \left(\varepsilon \frac{\partial f_{0}}{\partial \Delta \omega_{1}}+\varepsilon^{2} \frac{\partial f_{1}}{\partial \Delta \omega_{1}}+\cdots\right) \frac{d \Delta \omega_{1}}{d t}+\left(\varepsilon \frac{\partial f_{0}}{\partial \Delta \omega_{2}}+\varepsilon^{2} \frac{\partial f_{1}}{\partial \Delta \omega_{2}}+\cdots\right) \frac{d \Delta \omega_{2}}{d t} \\
& =\frac{-K_{31}}{2 H_{1}} \Delta \delta_{13}-\frac{K_{32}}{2 H_{1}} \Delta \delta_{23}-\frac{D_{3}}{2 H_{1}}\left(f_{0}+\varepsilon f_{1}+\cdots\right)
\end{aligned}
$$

The coefficients of the same order at both sides of Equation (19) shall be equal, so the power series can be expressed as

$$
\begin{gathered}
f_{0}=\frac{-K_{31} \Delta \delta_{13}-K_{32} \Delta \delta_{23}}{D_{3}} \\
f_{1}=\frac{2 \omega_{0} H_{1}\left(K_{31}+K_{32}\right)}{D_{3}^{2}} \Delta \omega_{1}+\frac{2 \omega_{0} H_{1}\left(K_{31}+K_{32}\right)}{D_{3}^{2}} \Delta \omega_{2} \\
+\frac{2 \omega_{0} H_{1} K_{31}\left(K_{31}+K_{32}\right)}{D_{3}^{3}} \Delta \delta_{13}+\frac{2 \omega_{0} H_{1} K_{32}\left(K_{31}+K_{32}\right)}{D_{3}^{3}} \Delta \delta_{23}
\end{gathered}
$$

Thus, the approximate manifold expression is

$$
\begin{aligned}
\Delta \omega_{3} & =f_{0}+\varepsilon f_{1}+o\left(\varepsilon^{2}\right) \cdots \approx\left[\frac{\omega_{0} K_{31}}{D_{3}^{3}}\left(K_{31}+K_{32}\right)-\frac{K_{31}}{D_{3}}\right] \Delta \delta_{13}+\left[\frac{2 H_{3} \omega_{0} K_{32}}{D_{3}^{3}}\left(K_{31}+K_{32}\right)-\frac{K_{32}}{D_{3}}\right] \Delta \delta_{23} \\
& +\frac{2 H_{3} \omega_{0}}{D_{3}^{2}}\left(K_{31}+K_{32}\right)\left(\Delta \omega_{1}+\Delta \omega_{2}\right) \\
& =a_{1} \Delta \delta_{13}+a_{2} \Delta \delta_{23}+a_{3} \Delta \omega_{1}+a_{3} \Delta \omega_{2}
\end{aligned}
$$

Substituting Equation (22) into Equation (13) yields

$$
\left[\begin{array}{c}
\Delta \dot{\delta}_{13} \\
\Delta \dot{\delta}_{23} \\
\Delta \dot{\omega}_{1} \\
\Delta \dot{\omega}_{2}
\end{array}\right]=\left[\begin{array}{cccc}
-a_{1} \omega_{0} & -a_{2} \omega_{0} & \left(1-a_{3}\right) \omega_{0} & \left(1-a_{3}\right) \omega_{0} \\
-a_{1} \omega_{0} & -a_{2} \omega_{0} & \left(1-a_{3}\right) \omega_{0} & \left(1-a_{3}\right) \omega_{0} \\
-\frac{K_{11}}{2 H_{1}} & \frac{K_{12}}{2 H_{1}} & -\frac{D_{1}}{2 H_{1}} & 0 \\
-\frac{K_{21}}{2 H_{2}} & -\frac{K_{22}}{2 H_{2}} & 0 & -\frac{D_{2}}{2 H_{2}}
\end{array}\right]\left[\begin{array}{c}
\Delta \delta_{13} \\
\Delta \delta_{23} \\
\Delta \omega_{1} \\
\Delta \omega_{2}
\end{array}\right]
$$

Taking the relative angular velocity offset $\Delta \omega_{12}$ as the state variable, Equation (23) can be written as

$$
\left[\begin{array}{c}
\Delta \dot{\delta}_{13} \\
\Delta \dot{\delta}_{23} \\
\Delta \dot{\omega}_{12}
\end{array}\right]=\left[\begin{array}{ccc}
-a_{1} \omega_{0} & -a_{2} \omega_{0} & 2 \omega_{0}\left(1-a_{3}\right) \\
-a_{1} \omega_{0} & -a_{2} \omega_{0} & 2 \omega_{0}\left(1-a_{3}\right) \\
\left(\frac{K_{31}}{2 H_{3}}-\frac{K_{11}}{2 H_{1}}\right) & \left(\frac{K_{12}}{2 H_{1}}+\frac{K_{32}}{2 H_{3}}\right) & \left(\frac{D_{2}}{2 H_{2}}-\frac{D_{1}}{2 H_{1}}\right)
\end{array}\right]\left[\begin{array}{c}
\Delta \delta_{13} \\
\Delta \delta_{23} \\
\Delta \omega_{12}
\end{array}\right]
$$

Then, the characteristic equation is given by

$$
p^{3}+\left(a_{2} \omega_{0}+a_{1} \omega_{0}+\frac{D_{1}}{2 H_{1}}-\frac{D_{2}}{2 H_{2}}\right) p^{2}+\left(a_{1} \omega_{0}+a_{2} \omega_{0}\right)\left(\frac{D_{1}}{2 H_{1}}-\frac{D_{2}}{2 H_{2}}\right) p-2 \omega_{0}\left(1-a_{3}\right)\left(\frac{K_{12}-K_{11}}{2 H_{1}}+\frac{K_{31}+K_{32}}{2 H_{3}}\right) p=0
$$


The eigenvalues of Equation (25) are calculated as

$$
\begin{gathered}
p_{1}=0 \\
\left\{\begin{array}{c}
p_{2,3}=\sigma \pm j \omega \\
\sigma=-\frac{1}{2}\left(a_{1} \omega_{0}+a_{2} \omega_{0}+\frac{D_{1}}{2 H_{1}}-\frac{D_{2}}{2 H_{2}}\right) \\
=-\frac{1}{2}\left[\frac{\omega_{0}^{2} K_{31}}{D_{3}^{2}}\left(K_{31}+K_{32}\right)-\frac{K_{31} \omega_{0}}{D_{3}}+\frac{2 H_{3} \omega_{0}^{2} K_{32}}{D_{3}^{3}}\left(K_{31}+K_{32}\right)-\frac{K_{32} \omega_{0}}{D_{3}}+\frac{D_{1}}{2 H_{1}}-\frac{D_{2}}{2 H_{2}}\right]
\end{array}\right.
\end{gathered}
$$

Finally, the expression of the dominant oscillation mode $\left(p_{2,3}\right)$ is mathematically deduced by the integral manifold theory, which indicates that two coherent groups of the sub-MGs swing against each other with the low damping ratio. Moreover, as seen from Equation (27), the dynamic performance of the dominant mode mainly depends on the negative real part $\sigma$. If oscillation mode $p_{2,3}$ moves further to the left half plane-namely, there is a decrease in $\sigma$-the ability to damp such oscillation becomes better. Therefore, the mechanism of influence of inertia on the small-signal stability of MG clusters is that reasonably adjusting the inertia of the sub-MGs (decreasing the inertia constant $H_{1}$ and increasing the inertia constants $\mathrm{H}_{2}$ and $\mathrm{H}_{3}$, in this case) is directly beneficial for improving the damping ratio of the dominant oscillation mode.

\subsection{Dynamic Inertia Constant Analysis of Three-Area PV-Based MG Clusters}

According to Equation (27), the virtual inertia control can provide both frequency support and oscillation damping. The regulation of the inertia constant in multi-area MG clusters for improving dynamic stability is an important issue. Therefore, the energy function of three-area system is constructed to further analyze the inertia constant.

Neglecting the effect of damping, the dynamic equation of three-area AC MG clusters can be written as

$$
\left\{\begin{array}{l}
2 H_{1} \frac{d \omega_{1}}{d t}=P_{m 1}-P_{e 1} \\
2 H_{2} \frac{d \omega_{2}}{d t}=P_{m 2}-P_{e 2} \\
2 H_{3} \frac{d \omega_{2}}{d t}=P_{m 3}-P_{e 3}
\end{array}\right.
$$

At steady state, the generators should maintain a balance between mechanical power and electromagnetic power. Under the initial operating conditions, the hypotheses are given by Equation (29)

$$
\left\{\begin{array}{l}
\delta_{12}=\delta_{0} \\
\delta_{13}=\delta_{0} \\
\delta_{23}=\delta_{0}
\end{array}\right.
$$

Then, the initial mechanical power of the equivalent three machine system can be given as

$$
\left\{\begin{array}{l}
P_{\mathrm{m} 1}=E_{1}^{2} G_{11}+E_{1} E_{2}\left(G_{12} \cos \delta_{0}+B_{12} \sin \delta_{0}\right)+E_{1} E_{3}\left(G_{13} \cos \delta_{0}+B_{13} \sin \delta_{0}\right) \\
P_{\mathrm{m} 2}=E_{2}^{2} G_{22}+E_{1} E_{2}\left(G_{12} \cos \delta_{0}-B_{12} \sin \delta_{0}\right)+E_{2} E_{3}\left(G_{23} \cos \delta_{0}+B_{23} \sin \delta_{0}\right) \\
P_{\mathrm{m} 3}=E_{3}^{2} G_{33}+E_{1} E_{3}\left(G_{13} \cos \delta_{0}-B_{13} \sin \delta_{0}\right)+E_{2} E_{3}\left(G_{23} \cos \delta_{0}-B_{23} \sin \delta_{0}\right)
\end{array}\right.
$$

In this study, the inductive MG is considered, thereby ignoring the line resistance. Substituting Equation (30) into Equation (28) yields

$$
\left\{\begin{array}{c}
2 H_{1} \frac{d \omega_{1}}{d t}=C_{12}\left(\sin \delta_{0}-\sin \delta_{12}\right)+C_{13}\left(\sin \delta_{0}-\sin \delta_{13}\right) \\
2 H_{2} \frac{d \omega_{2}}{d t}=C_{23}\left(\sin \delta_{0}-\sin \delta_{23}\right)-C_{12}\left(\sin \delta_{0}-\sin \delta_{12}\right) \\
2 H_{3} \frac{d \omega_{3}}{d t}=-C_{13}\left(\sin \delta_{0}-\sin \delta_{13}\right)-C_{23}\left(\sin \delta_{0}-\sin \delta_{23}\right) \\
C_{i j}=E_{i} E_{j} B_{i j}
\end{array}\right.
$$


Transforming Equation (31) yields

$$
\begin{aligned}
\omega_{12} \frac{d \omega_{12}}{d t}= & -\left(\frac{1}{2 H_{1}}+\frac{1}{2 H_{2}}\right) h \omega_{12}-\frac{1}{2 H_{1}} r \omega_{12}+\frac{1}{2 H_{2}} k \omega_{12} \\
\omega_{13} \frac{d \omega_{13}}{d t}= & -\left(\frac{1}{2 H_{1}}+\frac{1}{2 H_{3}}\right) r \omega_{13}-\frac{1}{2 H_{1}} h \omega_{13}-\frac{1}{2 H_{3}} k \omega_{13} \\
\omega_{23} \frac{d \omega_{23}}{d t}=- & -\left(\frac{1}{2 H_{2}}+\frac{1}{2 H_{3}}\right) k \omega_{23}+\frac{1}{2 H_{2}} h \omega_{23}-\frac{1}{2 H_{3}} r \omega_{23} \\
& \left\{\begin{array}{l}
C_{12}\left(\sin \delta_{12}-\sin \delta_{0}\right)=h \\
C_{13}\left(\sin \delta_{13}-\sin \delta_{0}\right)=r \\
C_{23}\left(\sin \delta_{23}-\sin \delta_{0}\right)=k
\end{array}\right.
\end{aligned}
$$

Multiplying Equations (33)-(35) by $H_{e q 1}, H_{e q 2}$ and $H_{e q 3}$ respectively, then adding them, yields

$$
\begin{aligned}
\left\{\begin{array}{l}
H_{e q 1}=\frac{2 H_{1} H_{2}}{H_{1}+H_{2}+H_{3}} \\
H_{e q 2}=\frac{2 H_{1} H_{3}}{H_{1}+H_{2}+H_{3}} \\
H_{e q 3}=\frac{2 H_{2} H_{3}}{H_{1}+H_{2}+H_{3}}
\end{array}\right. \\
\frac{2 H_{1} H_{2}}{H_{1}+H_{2}+H_{3}} \omega_{12} \frac{d \omega_{12}}{d t}+\frac{2 H_{1} H_{3}}{H_{1}+H_{2}+H_{3}} \omega_{13} \frac{d \omega_{13}}{d t}+\frac{2 H_{2} H_{3}}{H_{1}+H_{2}+H_{3}} \omega_{23} \frac{d \omega_{23}}{d t}=-h \omega_{12}-r \omega_{13}-k \omega_{23}
\end{aligned}
$$

Based on the concept of energy, the energy function of the equivalent three machine system can be constructed as

$$
\begin{gathered}
V=V_{k d}+V_{p} \\
=\frac{1}{2} H_{e q 1} \omega_{12}^{2}+\frac{1}{2} H_{e q 2} \omega_{13}^{2}+\frac{1}{2} H_{e q 3} \omega_{23}^{2}+C_{12}\left(\cos \delta_{0}-\cos \delta_{12}\right)-C_{12} \delta_{12} \sin \delta_{0}+C_{13}\left(\cos \delta_{0}-\cos \delta_{13}\right) \\
-C_{13} \delta_{13} \sin \delta_{0}+C_{23}\left(\cos \delta_{0}-\cos \delta_{23}\right)-C_{23} \delta_{23} \sin \delta_{0} \\
V_{k d}=\frac{1}{2} H_{e q 1} \omega_{12}^{2}+\frac{1}{2} H_{e q 2} \omega_{13}^{2}+\frac{1}{2} H_{e q 3} \omega_{23}^{2} \\
V_{p}=C_{12}\left(\cos \delta_{0}-\cos \delta_{12}\right)-C_{12} \delta_{12} \sin \delta_{0}+C_{13}\left(\cos \delta_{0}-\cos \delta_{13}\right)-C_{13} \delta_{13} \sin \delta_{0} \\
+C_{23}\left(\cos \delta_{0}-\cos \delta_{23}\right)-C_{23} \delta_{23} \sin \delta_{0}
\end{gathered}
$$

where, $V_{k d}$ is the kinetic energy of the system oscillation, which indicates the energy caused by the deviation between the speed of the generator and the reference speed of the system. $V_{p}$ is the potential energy caused by system oscillation.

According to Lyapunov's direct method, the value of the total energy must decrease monotonically to maintain the stability of the system.

$$
\frac{d V}{d t} \leq 0
$$

The derivative of Equation (39) is calculated as

$$
\begin{aligned}
\frac{d V}{d t} & =\left(\frac{1}{H_{1}+H_{2}+H_{3}}\right)^{2}\left(\omega_{12}^{2} H_{2}^{2}+\omega_{12}^{2} H_{2} H_{3}+\omega_{13}^{2} H_{3}^{2}+\omega_{13}^{2} H_{2} H_{3}-\omega_{23}^{2} H_{2} H_{3}\right) \frac{d H_{1}}{d t} \\
& +\left(\frac{1}{H_{1}+H_{2}+H_{3}}\right)^{2}\left(\omega_{12}^{2} H_{1}^{2}+\omega_{12}^{2} H_{1} H_{3}+\omega_{23}^{2} H_{3}^{2}+\omega_{23}^{2} H_{1} H_{3}-\omega_{13}^{2} H_{1} H_{3}\right) \frac{d H_{2}}{d t} \\
& +\left(\frac{1}{H_{1}+H_{2}+H_{3}}\right)^{2}\left(\omega_{13}^{2} H_{1}^{2}+\omega_{13}^{2} H_{1} H_{2}+\omega_{23}^{2} H_{2}^{2}+\omega_{23}^{2} H_{1} H_{2}-\omega_{12}^{2} H_{1} H_{2}\right) \frac{d H_{3}}{d t}
\end{aligned}
$$

To satisfy the Lyapunov stability criteria, combined with the analysis in Section 3.2, the inertia constant $H_{1}$ should be reduced and the inertia constants $H_{2}, \mathrm{H}_{3}$ should be increased. Meanwhile, the rate of inertia constant $\mathrm{H}_{1}$ decreases more than that of inertia constants $\mathrm{H}_{2}$ and $\mathrm{H}_{3}$. 
Based on the above mechanistic analysis in this section, the next vital problem naturally emerges: how to propose a reasonable and effective inertia control strategy for supercapacitors in order to improve the dynamic stability of MG clusters.

\section{Fuzzy-Based Dynamic Inertia Control of PV-Based MG Clusters}

\subsection{The Introduction of the Feedback Signal}

If the MPSC-based MG cluster is adopted in autonomous mode, it can be considered as a multi-area system. In the case of disturbances in MG clusters, it is assumed that regions 1 to $K$ are severely disturbed. The center of inertia is set as $K$, and the center of inertia of the remaining regions is set as $T-K$. Based on the concept of two machine equivalence, the equivalent angular velocity and power angle of two centers can be written as

$$
\begin{aligned}
& \left\{\begin{array}{l}
\widetilde{\omega}_{i}=\omega_{i}-\omega_{\mathrm{COI}} \\
\delta_{K}=\frac{\sum_{i=1}^{k} H_{i} \delta_{i}}{H_{K}} \\
\widetilde{\omega}_{K}=\frac{\sum_{i=1}^{k} H_{i} \widetilde{\omega}_{i}}{H_{K}} \\
H_{K}=\sum_{i=1}^{k} H_{i}
\end{array}\right. \\
& \left\{\begin{array}{l}
\delta_{T-K}=\frac{\sum_{i=k+1}^{n} H_{i} \delta_{i}}{H_{T-K}} \\
\widetilde{\omega}_{T-K}=\frac{\sum_{i=k+1}^{n} H_{i} \widetilde{\omega}_{i}}{H_{T-K}} \\
H_{T-K}=\sum_{i=k+1}^{n} H_{i}
\end{array}\right.
\end{aligned}
$$

The kinetic energy can be defined as

$$
V_{\mathrm{k}}=\frac{1}{2} H_{\mathrm{eq}} \omega_{\mathrm{eq}}^{2}
$$

The energy function, in center of inertia (COI) coordinates, is

$$
\begin{aligned}
V & =V_{\mathrm{k}}+V_{\mathrm{p}} \\
& =\frac{1}{2} H_{\mathrm{eq}} \omega_{\mathrm{eq}}^{2}+\left[\sum_{i=1}^{n} \int_{\delta_{s i}}^{\delta_{c i}}-\left(P_{m i}-P_{e i}-\frac{H_{i}}{H_{T}} P_{\mathrm{COI}}\right) d \delta_{i}\right]
\end{aligned}
$$

From Equation (47), it can be seen that if the value of the total energy remains constant, the change in kinetic energy is contrary to that in potential energy. Based on the analysis above, the value of the total energy must decrease monotonically to maintain the stability of the system. Thus, the equivalent angular velocity deviation can be introduced into the control method to adjust the active power of sub-MGs so as to improve the stability of the system.

\subsection{The Design of Fuzzy-Based Dynamic Inertia Controls}

Typically, as for MGs, the ESS is configured to smooth the power fluctuations generated by DGs. However, for MGs in autonomous mode, the ESS is required to participate in the voltage and frequency control of the MGs. In this study, the attribute of the supercapacitor is exploited to generate a virtual inertia response for the dynamic stability of MG clusters.

The power extracted from a supercapacitor can be computed as follows: 


$$
\Delta P_{c}=\frac{C U_{c N}^{2} d \rho_{s o c}}{2 d t}=K \frac{C U_{c N}^{2} d \omega_{e}}{2 d t}=K \frac{C U_{c N}^{2} d f}{2 d t}
$$

It can be seen that by changing the virtual inertia coefficient $K$ in traditional virtual inertia control, the corresponding inertia support can be provided to guarantee the smooth transition of the system through the disturbance process. However, the relationship between the power of supercapacitor $P_{c}$ and the synchronous angular speed $\omega_{e}$ is non-linear in nature. The fuzzy logic control method has been widely used in real-time industrial applications. Due to its high robustness and stability, a fuzzy logic controller can offer better control performance than classical controllers (e.g., PID controllers). In this study, fuzzy-based dynamic inertia control is applied to the supercapacitor, thereby supplying a rapid inertia adjustment of the system.

In this study, the two-dimensional fuzzy controller was designed as shown in Figure 4. The design process for a fuzzy-based dynamic controller is as follows:

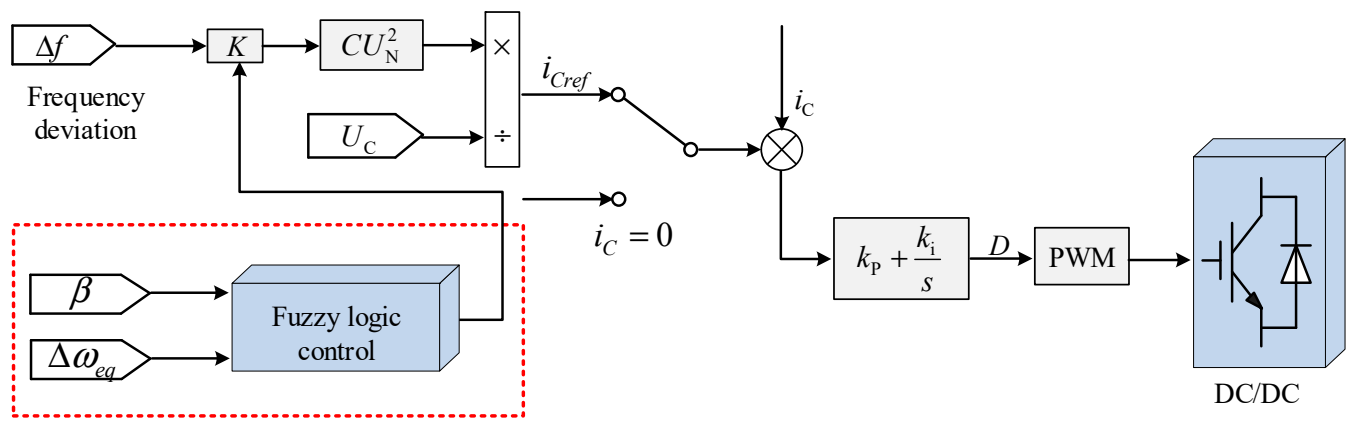

Figure 4. The fuzzy-based dynamic control strategy.

(a) Selecting input variables and membership functions

Two input variables are the relative angular velocity deviation of the inertia center $\Delta \omega_{e q}$ and $\beta$. The relative angular velocity deviation of the inertia center can be obtained by using the wide area measurement system (WAMS) in actual practice. $\beta$ is calculated as

$$
\beta=\arctan \frac{d \Delta \omega_{e q} / d t}{\Delta \omega_{e q}}, \beta \in\left[-\frac{\pi}{2}, \frac{\pi}{2}\right]
$$

The universe of discourse of input variable $\beta$ is $\mathrm{P}$ and $\mathrm{N}$. By distinguishing the positive and negative values of $\beta$, the process of energy change can be identified. The universe of discourse of the relative angular velocity deviation of inertia center $\Delta \omega_{\text {eq }}$ and output variable are NB, NM, NS, ZE, PS, PM and PB. Additionally, the membership functions (shown in Figure 5) of input variables are trimf and gbellmf.

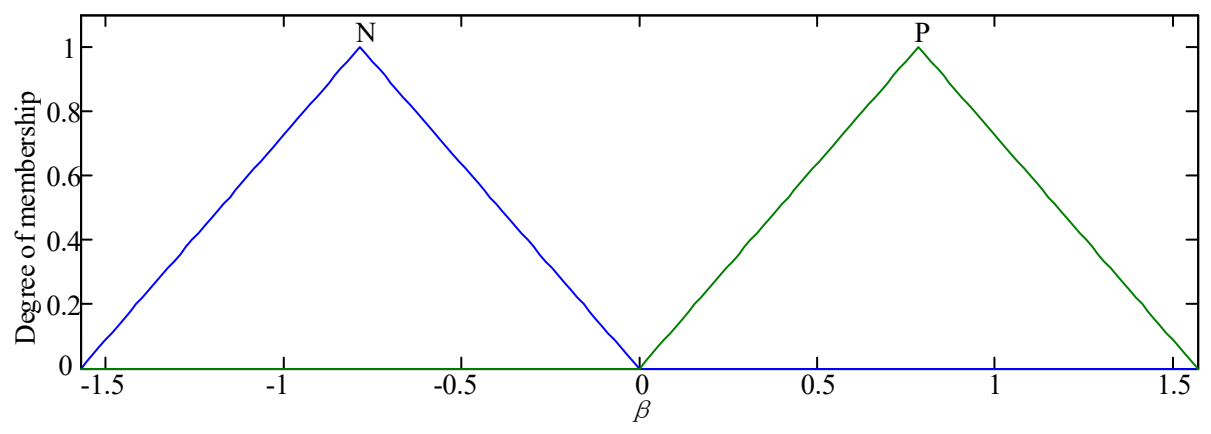

(a)

Figure 5. Cont. 


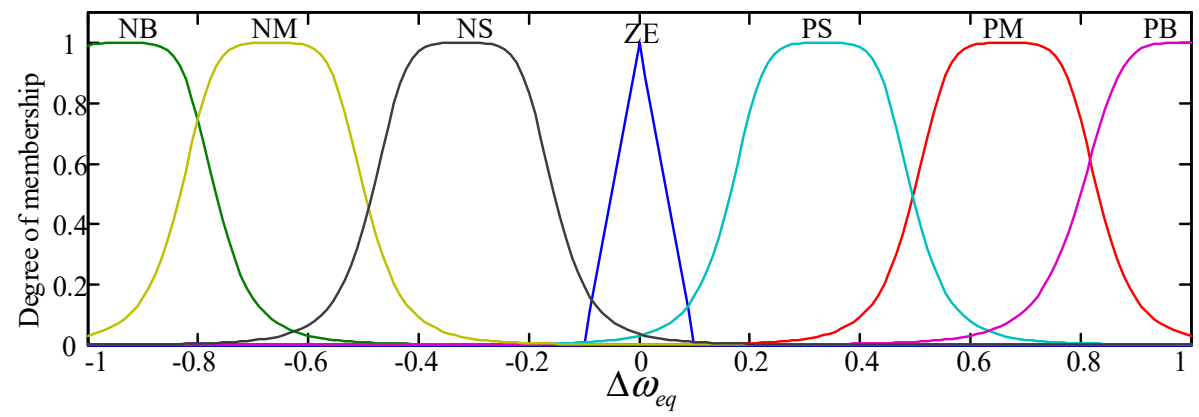

(b)

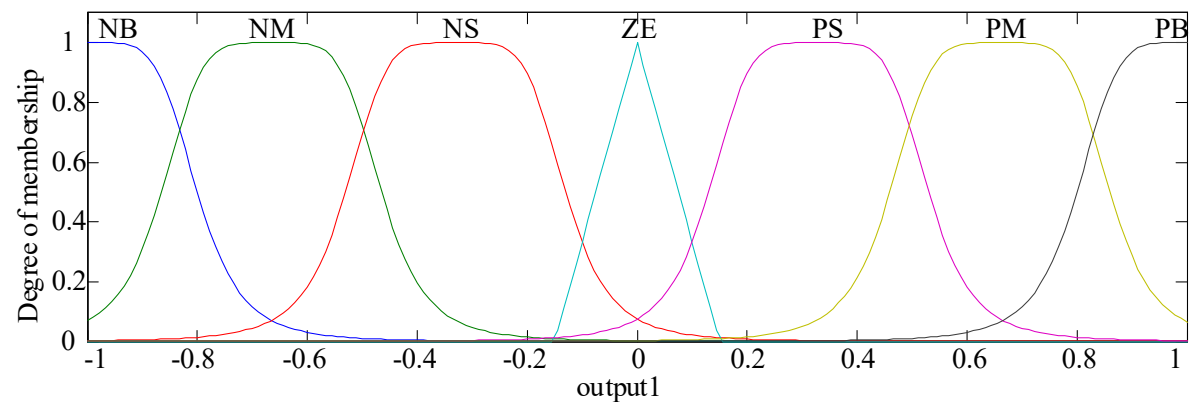

(c)

Figure 5. Membership functions: (a) the membership function of $\beta$, (b) the membership function of $\Delta \omega_{e q}$ and (c) the membership function of the output.

(b) Establishing fuzzy rules

Generally, fuzzy rules need to be built based on the control principle and practical experience. In this study, the control principle is to adjust the charge and discharge current of a supercapacitor as the angular frequency deviation of inertia changes. The specific fuzzy rules are listed in Table 1.

Table 1. Fuzzy rules table.

\begin{tabular}{cccccccc}
\hline \multirow{2}{*}{$\beta$} & \multicolumn{7}{c}{$\Delta \omega_{\text {eq }}$} \\
\cline { 2 - 7 } & NB & NM & NS & ZE & PS & PM & PB \\
\hline P & PB & PM & PS & ZE & NS & NM & NB \\
N & NB & NM & NS & ZE & PS & PM & PB \\
\hline
\end{tabular}

(c) Fuzzy inference system and defuzzification

The fuzzy-based dynamic controller designed in this study adopts Mamdani's fuzzy inference method. The defuzzification method is the centroid calculation.

\subsection{A Discussion of Fuzzy-Based Dynamic Inertia Control}

The fuzzy-based dynamic inertia control strategy is shown in Figure 4. Due to the reduction of the frequency response and regulation capability, severe frequency or power fluctuations may occur under short circuit, loads or irradiation disturbances. In this study, each of the sub-MGs in MG clusters is equipped with the fuzzy-based dynamic inertia control. In order to avoid the overcharge and discharge of supercapacitors, the operation mode of the supercapacitor is also designed in this study. In the process of charging, the SOC above $90 \%$ is defined as the low inertia region. In the process of discharging, the SOC below $10 \%$ is defined as the low inertia region. 
The proposed control process is summarized as follows: when the loads in a sub-MG change, it may lead to a sudden change of frequency. Firstly, the SOC of the supercapacitor is detected to determine the operating area. If the supercapacitor in this sub-MG is located in the low inertia region, the current of this supercapacitor will be set to zero, so that the supercapacitor no longer provides inertial support. Meanwhile, the supercapacitors in other interconnected sub-MGs are triggered to provide inertia support. If the supercapacitor in this sub-MG is located in the normal working region, the input signals $\beta$ and $\Delta \omega_{e q}$ are delivered to the fuzzy controller and then the virtual inertia coefficient $K$ is evaluated along with the change in the relative angular velocity deviation of the inertia center. Through the dynamic adjustment of the inertia coefficient, the current of the supercapacitor can be controlled to provide instantaneous energy; thereby, a large virtual inertia $H$ is emulated to reduce the frequency deviation. During the frequency recovery period, the virtual inertia is reduced gradually to avoid potential power oscillation. Similarly, in terms of short circuit faults by detecting the signal $\beta$ and $\Delta \omega_{e q}$, the fuzzy-based dynamic inertia controller adjusts the value of virtual inertia $H$ in real time to suppress power oscillation.

\section{Simulation Studies and Discussion}

In order to investigate the preceding theoretical analysis presented in Section 3 and the performance of the fuzzy-based dynamic inertia control strategy, the three-area PV-based MG clusters in autonomous mode are considered, as shown in Figure 6. The three-area PV-based MG clusters consist of six microturbines $\left(G_{1}, G_{2}, G_{3}, G_{4}, G_{5}\right.$ and $\left.G_{6}\right)$, three $P V$ arrays $\left(P V_{1}, P V_{2}\right.$ and $\left.P V_{3}\right)$, three supercapacitors and three aggregated loads $\left(\mathrm{L}_{1}, \mathrm{~L}_{2}\right.$ and $\left.\mathrm{L}_{3}\right)$. As shown, $\mathrm{G}_{1}, \mathrm{G}_{2}, \mathrm{PV}_{1}$ and one supercapacitor constitute MG1; MG2 contains $\mathrm{G}_{3}, \mathrm{G}_{4}, \mathrm{PV}_{2}$ and another supercapacitor; and MG3 has the same structure. In the simulation studies, the PV arrays, microturbines, supercapacitors and DC/DC converters use detailed circuit models. The DC/AC inverter uses the averaged model. The main parameters of the three-area PV-based MG clusters are given in Tables 2-4. The simulation results for different case studies are obtained using MATLAB/Simulink to verify the proposed schemes.

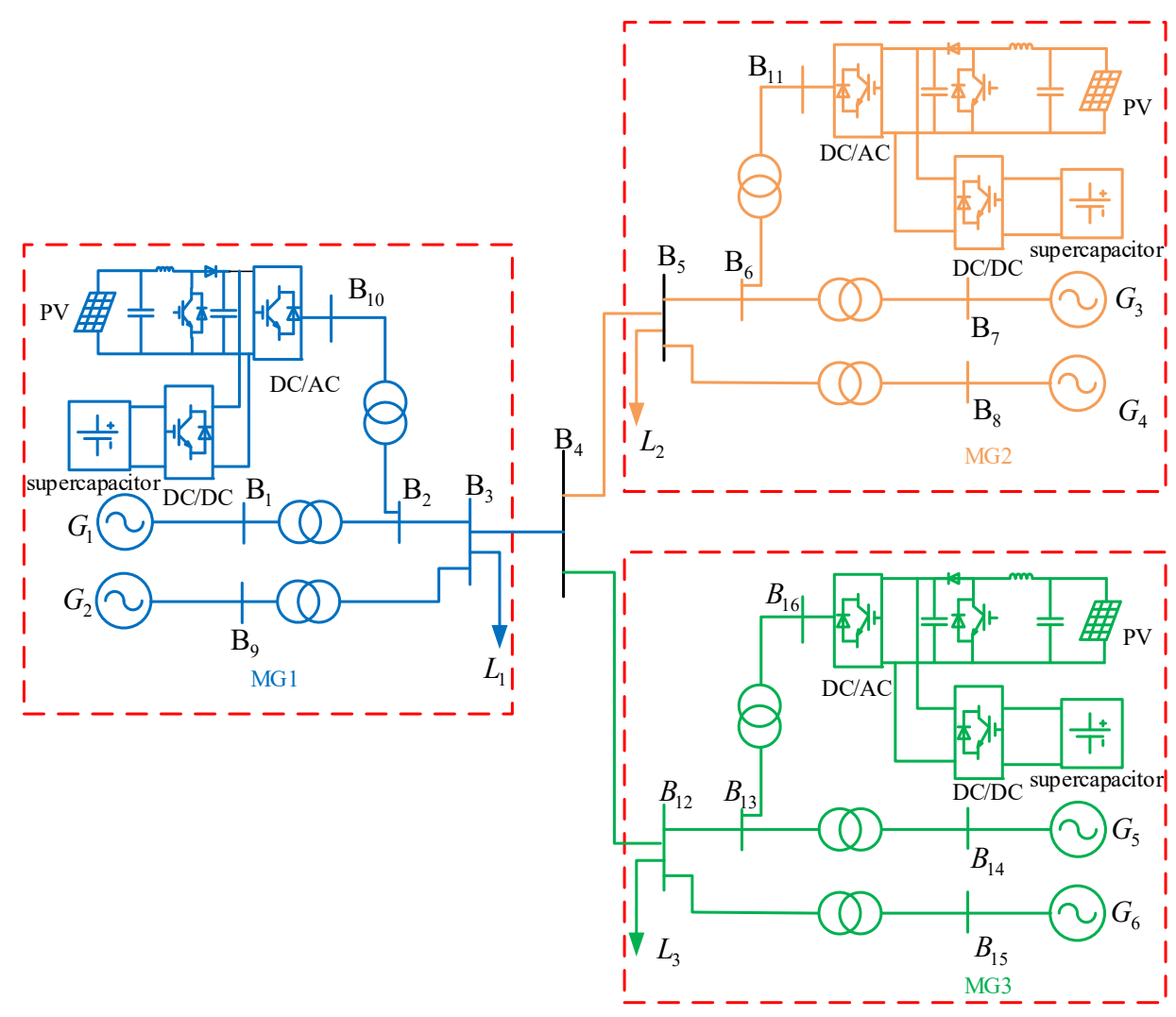

Figure 6. The structure of the three-area islanded MGs system. 
Table 2. PV array parameters (SunPower SPR-305-WHT).

\begin{tabular}{ccc}
\hline Parameter & Symbol & Value \\
\hline Number of cells per module & $N_{\mathrm{c}}$ & 96 \\
Number of series-connected modules per string & $N_{\mathrm{s}}$ & 5 \\
Number of parallel strings & $N_{\mathrm{p}}$ & 66 \\
Photo-generated current & $I_{\mathrm{ph}}$ & $5.9602 \mathrm{~A}$ \\
Diode saturation current & $I_{\mathrm{sat}}$ & $1.1753 \times 10^{-8} \mathrm{~A}$ \\
Electric charge & $q$ & $1.6 \times 10^{-19} \mathrm{C}$ \\
Boltzmann constant & $\mathrm{K}$ & $1.38 \times 10^{-23} \mathrm{~J} / \mathrm{K}$ \\
\hline
\end{tabular}

Table 3. Microturbine parameters.

\begin{tabular}{ccc}
\hline Parameter & Symbol & Value \\
\hline Microturbine in MG1 & & \\
\hline Rated power & $S$ & $200 \mathrm{kVA}$ \\
d-axis synchronous reactance & $X_{\mathrm{d}}$ & 2 \\
d-axis synchronous transient reactance & $X_{\mathrm{d}}^{\prime}$ & 0.35 \\
d-axis synchronous subtransient reactance & $X_{\mathrm{d}}{ }^{\prime \prime}$ & 0.252 \\
q-axis synchronous reactance & $X_{\mathrm{q}}$ & 1.9 \\
q-axis synchronous transient reactance & $X_{\mathrm{q}}^{\prime}$ & 0.55 \\
q-axis synchronous subtransient reactance & $X_{\mathrm{q}}{ }^{\prime \prime}$ & 0.243 \\
\hline Microturbine in MG2 and MG3 & \\
\hline Rated power & $S$ & $100 \mathrm{kVA}$ \\
d-axis synchronous reactance & $X_{\mathrm{d}}$ & 2.13 \\
d-axis synchronous transient reactance & $X_{\mathrm{d}}{ }^{\prime}$ & 0.308 \\
d-axis synchronous subtransient reactance & $X_{\mathrm{d}}{ }^{\prime}$ & 0.234 \\
q-axis synchronous reactance & $X_{\mathrm{q}}$ & 2.07 \\
q-axis synchronous transient reactance & $X_{\mathrm{q}}^{\prime}$ & 0.906 \\
q-axis synchronous subtransient reactance & $X_{\mathrm{q}}^{\prime \prime}$ & 0.234 \\
\hline
\end{tabular}

Table 4. MG parameters.

\begin{tabular}{cc}
\hline Parameter & Value \\
\hline Rated power of $\mathrm{PV}_{1}, \mathrm{PV}_{2}$ and $\mathrm{PV}_{3}$ & $100 \mathrm{~kW}$ \\
Rated power of $\mathrm{G}_{1}$ and $\mathrm{G}_{2}$ & $200 \mathrm{kVA}$ \\
Rated power of $\mathrm{G}_{3}, \mathrm{G}_{4}, \mathrm{G}_{5}$ and $\mathrm{G}_{6}$ & $100 \mathrm{kVA}$ \\
$\mathrm{DC}$ voltage & $500 \mathrm{~V}$ \\
Nominal frequency & $50 \mathrm{~Hz}$ \\
Load 1 & $320 \mathrm{~kW}$ \\
Load 2 & $160 \mathrm{~kW}$ \\
Load 3 & $240 \mathrm{~kW}$ \\
DC/DC Converter & \\
\hline Capacitor & $1.2 \mathrm{mF}$ \\
Inductor $(L)$ & $5 \mathrm{mH}$ \\
\hline
\end{tabular}

\subsection{Inertia Responses for Short Circuit Faults}

\subsubsection{The Effectiveness of the Fuzzy-Based Dynamic Control Method}

To illustrate the effect of the virtual inertia from the supercapacitor on the system's dynamic stability, the following four cases are considered:

- $\quad$ Case A: three MGs without the inertia control;

- $\quad$ Case B: MG1 with the fuzzy-based dynamic control, other MGs without control;

- $\quad$ Case C: MG2 with the fuzzy-based dynamic control, other MGs without control; 
- Case D: MG3 with the fuzzy-based dynamic control, other MGs without control.

A $0.1 \mathrm{~s}$ three-phase short circuit fault at Bus B4 was applied. The PV operates at the maximum power point, and the irradiance is assumed to be $800 \mathrm{~W} / \mathrm{m}^{2}$. As shown in Figure $7 \mathrm{a}$, after fault clearance, a sustained power oscillation in the low inertia system can be observed due to the poor damping of the interconnected PV MG clusters. In Case B, the fuzzy-based dynamic inertia controller of the supercapacitor in MG1 is activated to provide active power support. In Figure 7a-c, the oscillation amplitude of $\mathrm{G}_{1}$ 's active power is significantly reduced, and the duration is also shortened to $10.5 \mathrm{~s}$. During the recovery of power oscillation, the power of the supercapacitor in MG1 is continuously changing to provide variable virtual inertia, so as to improve the system's oscillating characteristics. As the SOC of the supercapacitor is above $90 \%$ or below $10 \%$, the supercapacitor in MG1 is out of action, but the supercapacitor in MG2 can also damp the power oscillation. In Case C, as shown in Figure 7d,e, the supercapacitor in MG2 uses variable inertia to provide additional positive damping. In Figure 7a, the duration of power oscillation is reduced to $11.5 \mathrm{~s}$. It is indicated that the supercapacitor also has an effect on power oscillation. In Case D, when the fuzzy-based dynamic inertia controller is applied to MG3, the supercapacitor in MG3 can use its energy to provide support for the MG clusters at the beginning of power fluctuation. From the results, it is concluded that the fuzzy-based dynamic inertia control can improve the small-signal stability of MG clusters in the autonomous mode.

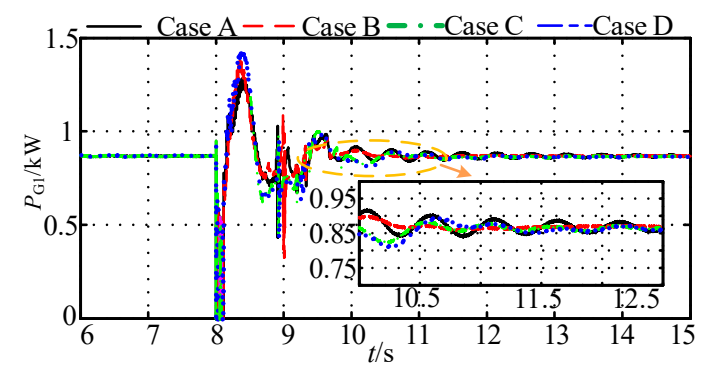

(a)

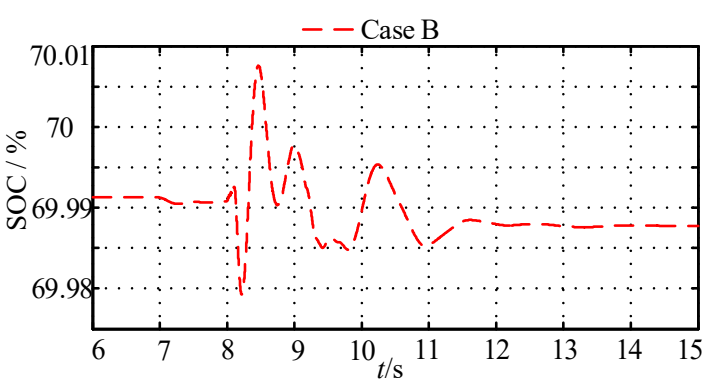

(b)

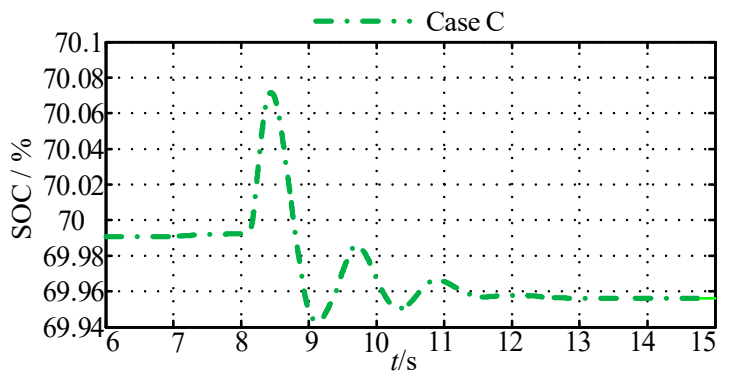

(d)

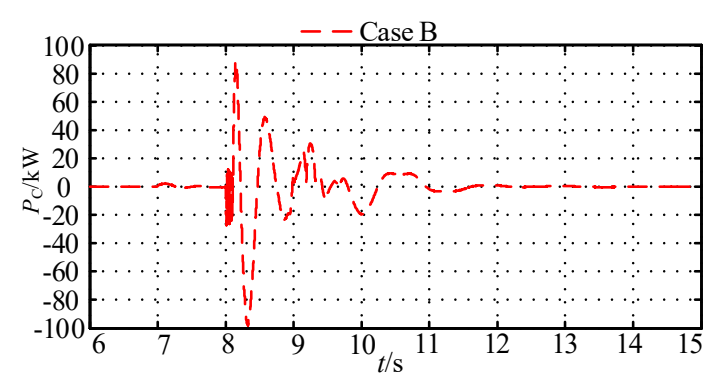

(c)

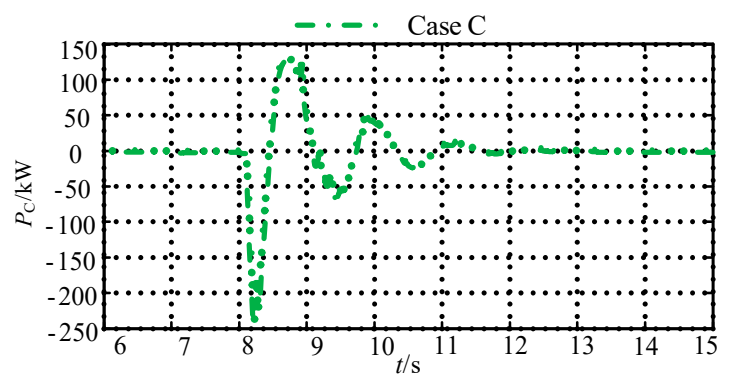

(e)

Figure 7. Cont. 


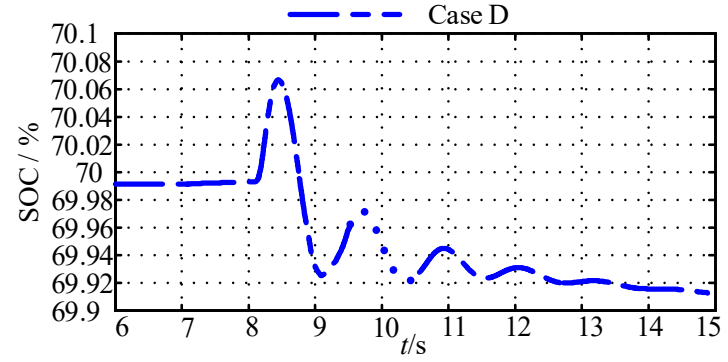

(f)

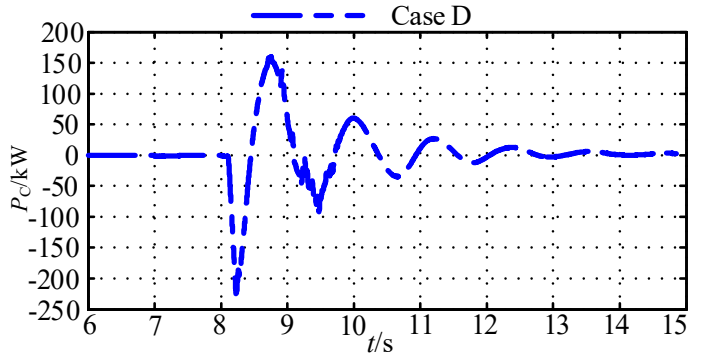

(g)

Figure 7. The dynamic response of the MG clusters after short circuit faults: (a) the active power of $\mathrm{G}_{1}$, (b) the state of charge (SOC) of the supercapacitor of MG1, (c) the power of the supercapacitor of MG1, (d) the SOC of the supercapacitor of MG2, (e) the power of the supercapacitor of MG2, (f) the SOC of the supercapacitor of MG3, and (g) the power of the supercapacitor of MG3.

\subsubsection{The Advantages of the Fuzzy-Based Dynamic Control Method}

To further illustrate the advantages of the fuzzy-based dynamic control for system damping, the experimental results are compared for the three cases:

- Case A: three MGs without inertia control;

- Case B: MG1 with the fuzzy-based dynamic control, other MGs without control;

- Case E: MG1 with the additional droop control, other MGs without control.

Figure 8 shows the comparison between the fuzzy-based dynamic inertia control and the additional droop control. As shown in Figure 8a, power oscillations in $\mathrm{G}_{1}$ are significantly reduced by using the fuzzy-based dynamic inertia control method. Compared with Case $E$, the amplitude of $G_{1}$ 's power oscillation is much lower, and its duration is decreased from around $12.5 \mathrm{~s}$ to $10.5 \mathrm{~s}$. This indicates that the active power of the supercapacitor generated by the fuzzy-based dynamic inertia control can better damp the power oscillation. Therefore, the fuzzy-based dynamic inertia control method performs better in terms of its effect on the oscillating characteristics of MG clusters.

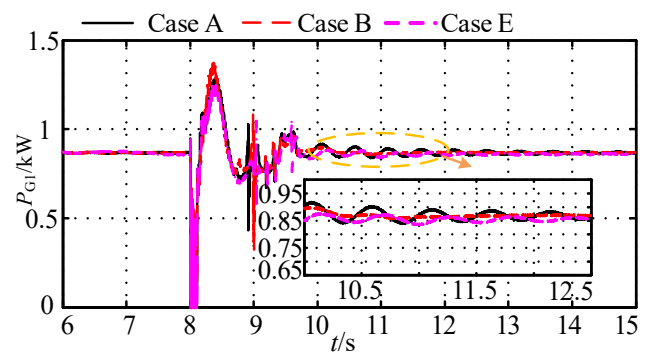

(a)

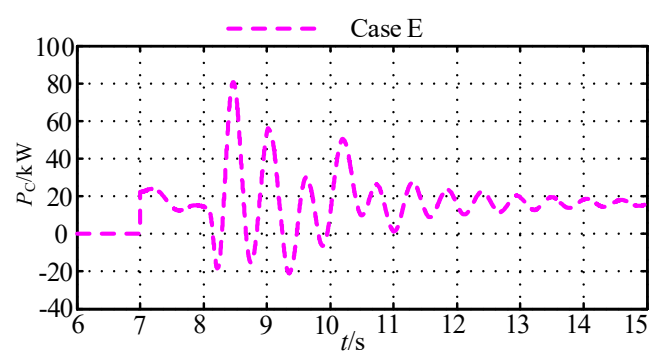

(b)

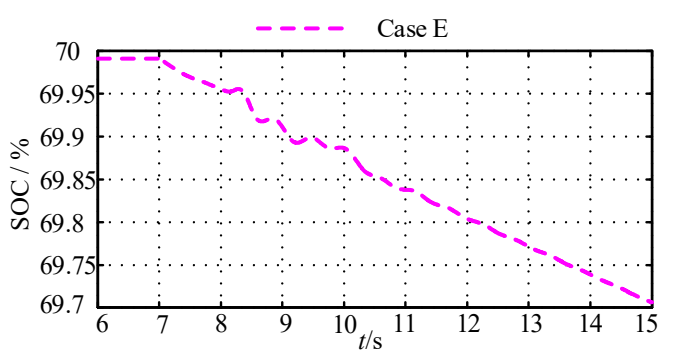

(c)

Figure 8. Comparison of dynamic responses after short circuit faults: (a) the active power of $G_{1}$, (b) the power of the supercapacitor of MG1 in Case E, and (c) the SOC of the supercapacitor of MG1 in Case E. 


\subsection{Inertia Responses Under Sudden Load Changes}

\subsubsection{The Effectiveness of the Fuzzy-Based Dynamic Control Method}

In this study, the irradiance is set as $800 \mathrm{~W} / \mathrm{m}^{2}$ and the PV in each sub-MG operates at the maximum power point. During the test, load L1 suddenly increases by $100 \mathrm{~kW}$ at $7 \mathrm{~s}$, causing a temporary fall in the system frequency. Without inertia control, as shown in Figure 9a, a large frequency drop of $0.38 \mathrm{~Hz}$ can be observed due to the small inertia. Additionally, from Figure $9 \mathrm{~b}-\mathrm{d}$, it can be observed that the active power of the microturbines in the three-area MGs are increased when the load in MG1 suddenly increases. In Case B, it can be seen from Figure 9a that the minimum system frequency is increased from $49.62 \mathrm{~Hz}$ to $49.83 \mathrm{~Hz}$ and that the frequency change rate is also decreased by $55.2 \%$. In Figure 9e,f, the supercapacitor in MG1 uses fuzzy-based dynamic inertia control to extract the stored energy, so as to improve the frequency stability. Due to the power provided by the supercapacitor, the rise in the active power of the microturbines is alleviated. In Case C, fuzzy-based dynamic control is applied to the supercapacitor in MG2. As shown in Figure 9a, the minimum system frequency is increased from $49.62 \mathrm{~Hz}$ to $49.79 \mathrm{~Hz}$, and the frequency change rate is decreased by $44.7 \%$. As seen in Figure $9 g, h$, the active power of the supercapacitor increases to compensate for the imbalance in power. This demonstrates that the supercapacitor in MG2 can also improve the frequency stability caused by the mutation load in MG1. Similarly, when the supercapacitors in MG1 or MG2 are located in the low inertia region, as shown in Figure 9i,j, the supercapacitor in MG3 with fuzzy-based dynamic inertia control can also regulate its energy to increase the inertia of the MG and adjust the dynamic frequency response. Hence, it is concluded that the supercapacitor can mimic the rotational inertia of conventional generators, which can enhance the frequency stability.

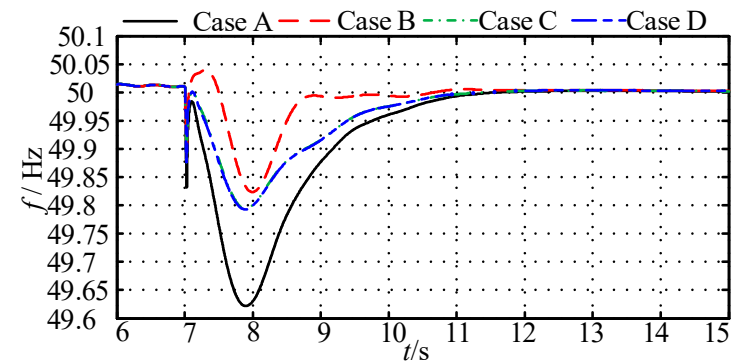

(a)

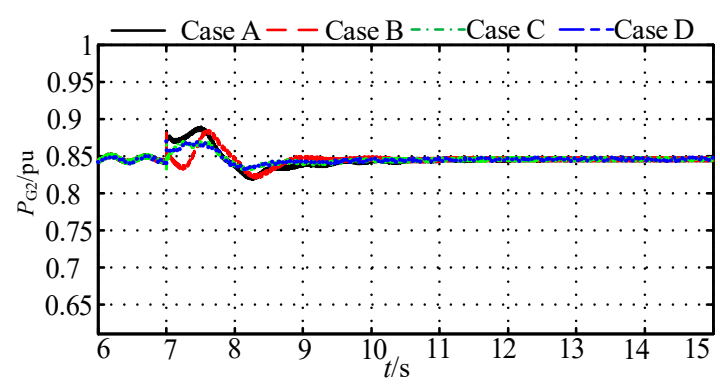

(c)

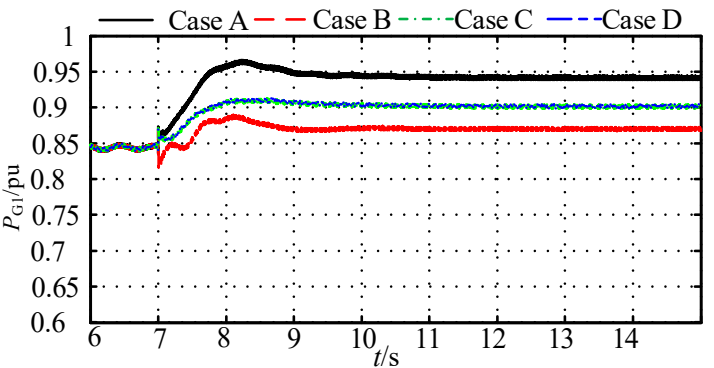

(b)

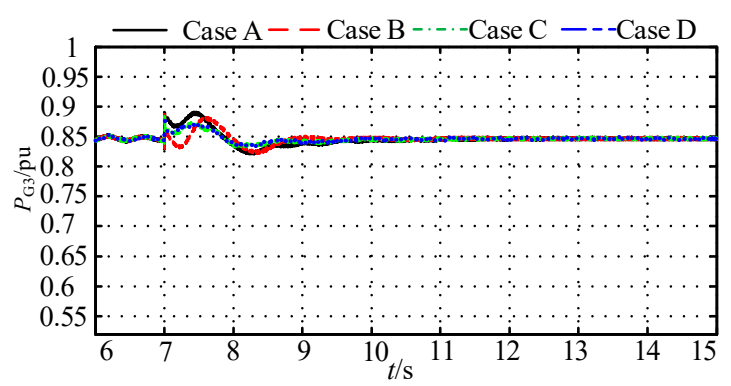

(d)

Figure 9. Cont. 


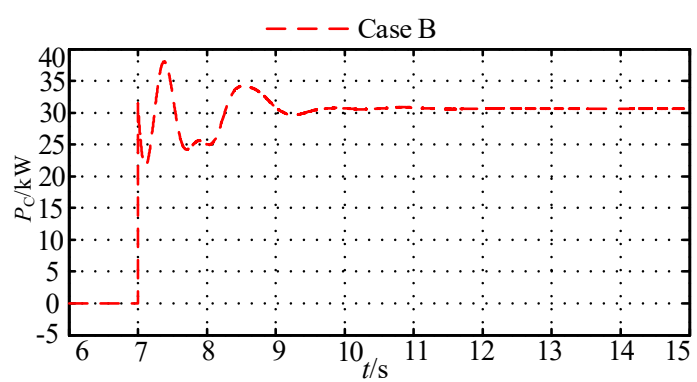

(e)

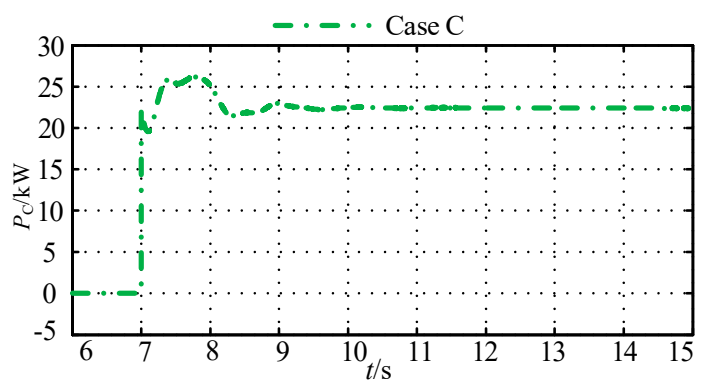

(g)

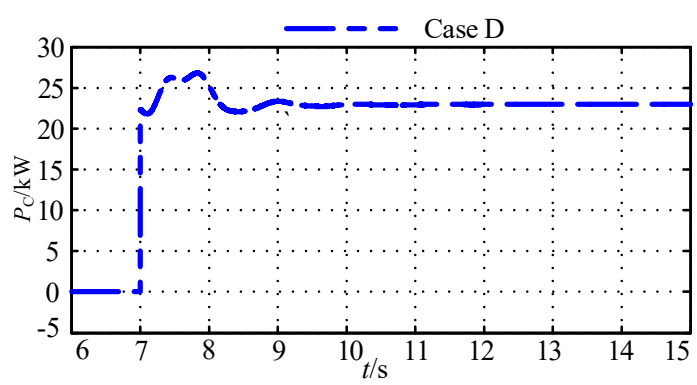

(i)

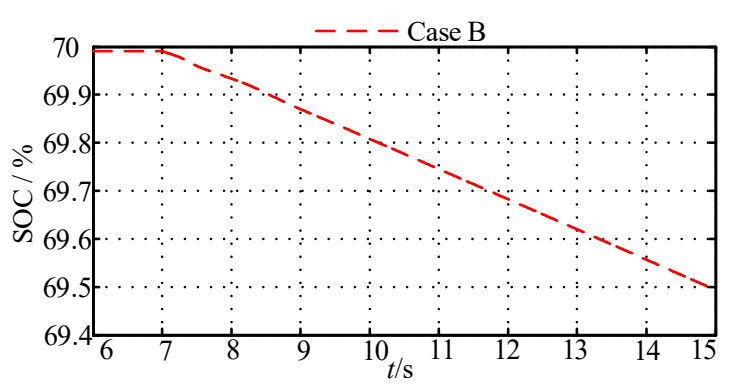

(f)

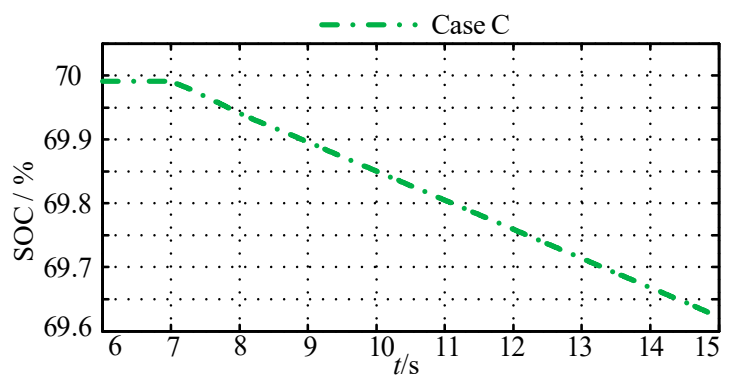

(h)

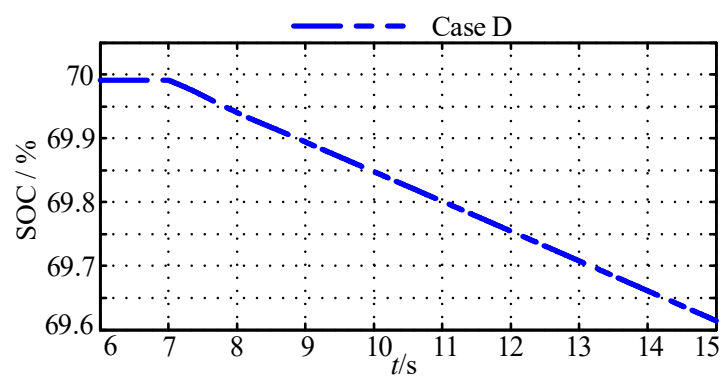

(j)

Figure 9. The dynamic response of the MG clusters during a sudden increase in load L1 by $100 \mathrm{~kW}$ : (a) the system frequency, (b) the active power of $G_{1},(\mathbf{c})$ the active power of $G_{2},(d)$ the active power of $G_{3}$, (e) the power of the supercapacitor of MG1, (f) the SOC of the supercapacitor of MG1, (g) the power of the supercapacitor of MG2, (h) the SOC of the supercapacitor of MG2, (i) the power of the supercapacitor of MG3, and (j) the SOC of the supercapacitor of MG3.

\subsubsection{The Advantages of the Fuzzy-Based Dynamic Control Method}

To further investigate the advantages of the fuzzy-based dynamic control method for frequency stability, the experimental results are compared for the three cases:

- $\quad$ Case A: three MGs without inertia control;

- $\quad$ Case B: MG1 with the fuzzy-based dynamic control, other MGs without control;

- $\quad$ Case E: MG1 with the additional droop control, other MGs without control.

Figure 10 compares the performance in Case B and Case E. Compared with Case A, both of the two inertia control schemes make the frequency nadir, and the change rate reduces. Moreover, fuzzy-based dynamic inertia control causes a smaller frequency drop and better recovery. The frequency drop is reduced from $0.2 \mathrm{~Hz}$ to $0.17 \mathrm{~Hz}$. Hence, compared with the additional droop control, the frequency drop and fluctuation subsequent to sudden load change can be better alleviated through using fuzzy-based dynamic inertia control. It is concluded that the fuzzy-based dynamic inertia control method can better contribute to frequency stability. 


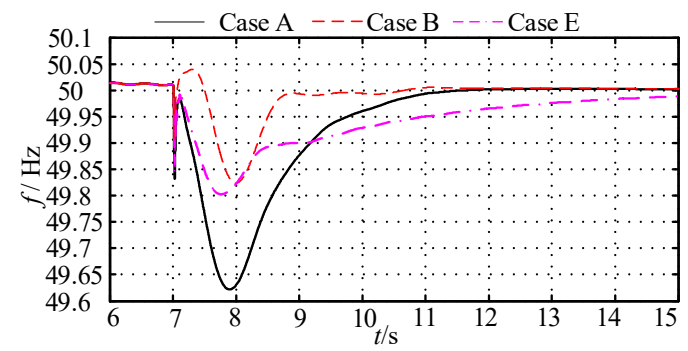

(a)

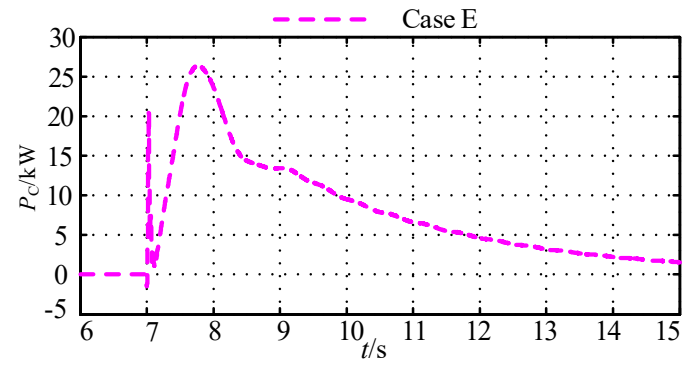

(b)

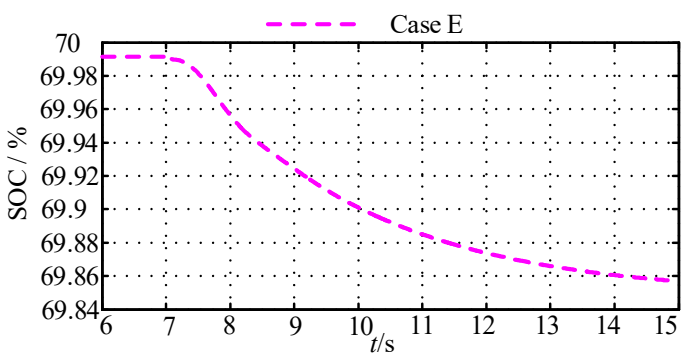

(c)

Figure 10. Comparison of dynamic responses under sudden load increase: (a) the system frequency, (b) the power of the supercapacitor of MG1 in Case E, and (c) the SOC of the supercapacitor of MG1 in Case E.

\subsection{Inertia Responses Under the Real Sun Condtion}

In this testing scenario, the irradiance variation of the PV in MG1 is shown in Figure 11a. The real sun condition is mainly affected by irradiance. To verify the robustness and effectiveness of fuzzy-based dynamic inertia control, it is assumed that the irradiance of the PV in MG1 is decreasing at $t=6 \mathrm{~s}$ and increasing at $t=9 \mathrm{~s}$. Because of the fluctuation of irradiance, the output power of the PV in MG1 is changing, leading to a power imbalance in the system. Then, the power imbalance induces a change in the system frequency. Without inertia control, as shown in Figure 11b, a significant fall in the system frequency can be observed due to the small inertia. In Case B, as shown in Figure 11c,d, the fuzzy-based dynamic inertia control is added in MG1, and then the fall in the amplitude of the frequency decreases. Similarly, as shown in Figure 11e,h, when the supercapacitors in MG1 or MG2 are located in the low inertia region, a supercapacitor in another sub-MG with fuzzy-based dynamic inertia control can also regulate its energy to increase the inertia of the MG.

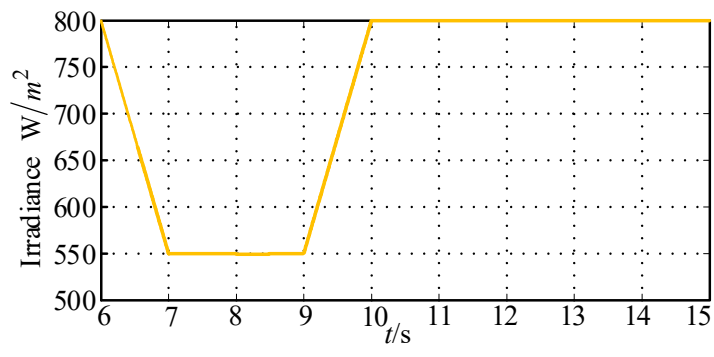

(a)

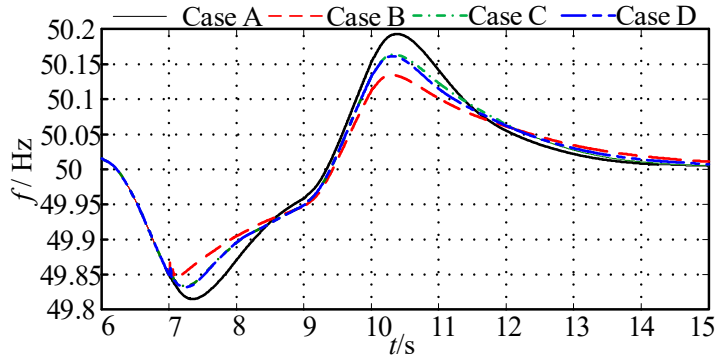

(b)

Figure 11. Cont. 


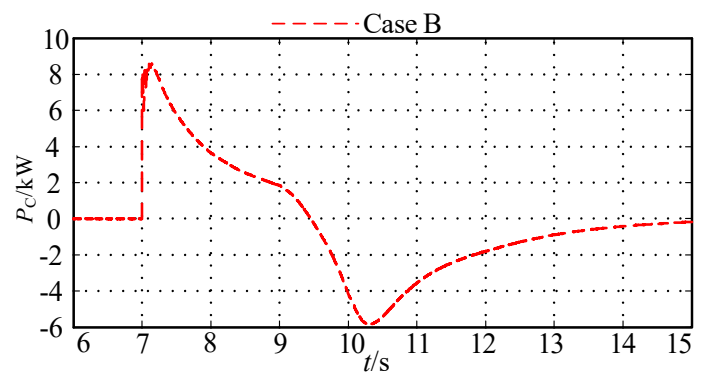

(c)

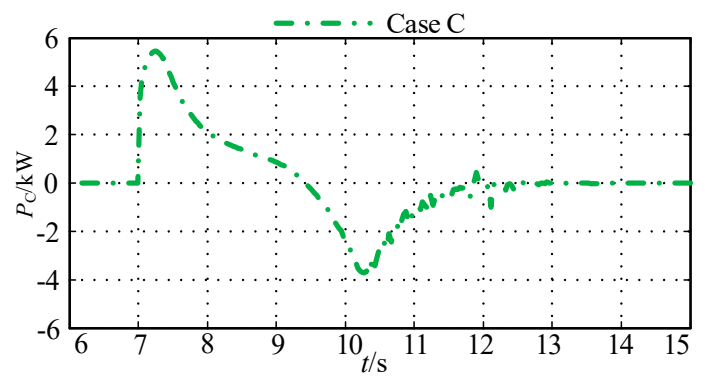

(e)

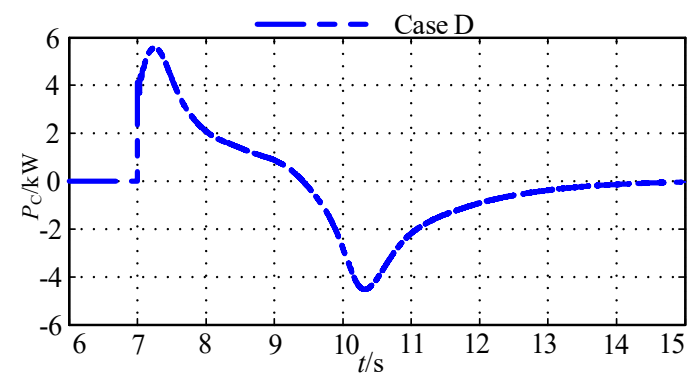

(g)

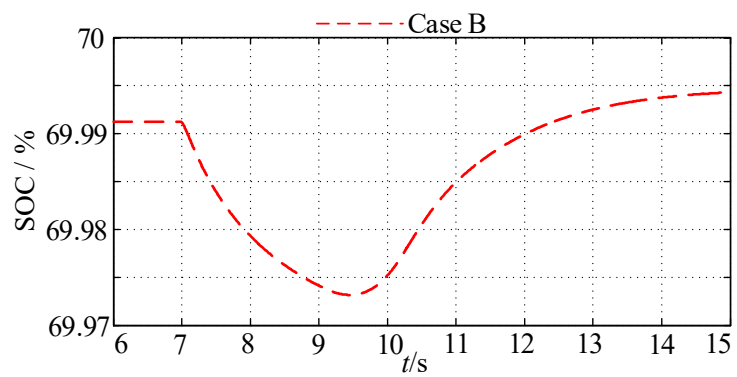

(d)

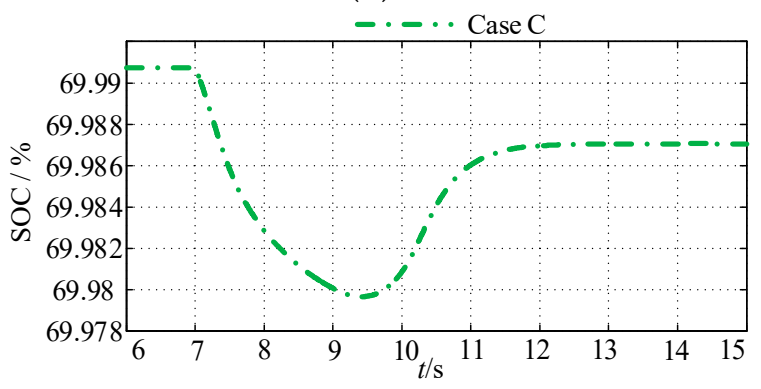

(f)

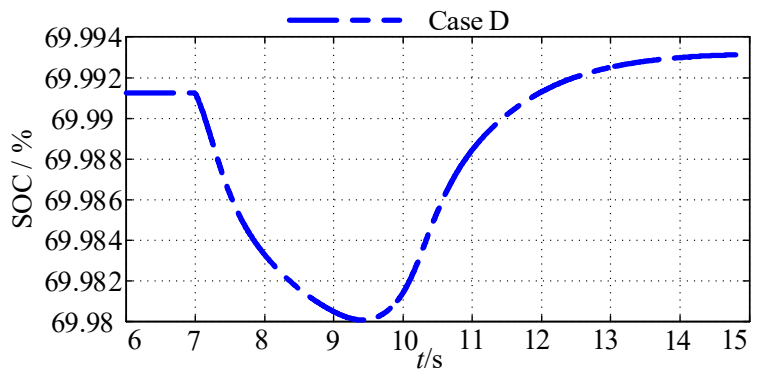

(h)

Figure 11. The dynamic response of the MG clusters under irradiance variation: (a) the irradiance of the PV in MG1, (b) the system frequency, (c) the power of the supercapacitor of MG1, (d) the SOC of the supercapacitor of MG1, (e) the power of the supercapacitor of MG2, (f) the SOC of the supercapacitor of MG2, (g) the power of the supercapacitor of MG3, and (h) the SOC of the supercapacitor of MG3.

\section{Conclusions}

This study aims to deal with the dynamic stability of multi-area MG clusters when the autonomous mode is adopted. The mechanistic analysis in this study concludes that dynamic inertia control for supercapacitors is significantly helpful for improving the frequency stability and suppressing power oscillations in MG clusters. Moreover, the fuzzy-based dynamic inertia control strategy is proposed, where designed controllers are able to offer better performance by self-tuning gains. Then, through simulation under various scenarios, the effectiveness and advantages of the fuzzy-based dynamic inertia control are also verified. Therefore, the fuzzy-based dynamic inertia control proposed in this paper can more effectively improve the dynamic stability of multi-area MG clusters in comparison with the additional droop control scheme.

As for the multi-area MG clusters in autonomous mode, the integral manifold theory used in this study can provide fundamental theoretical support to analyze the dynamic stability of multi-area MG clusters in the future. Meanwhile, the fuzzy-based dynamic inertia control method can offer new inspiration to improve the dynamic stability of multi-area MG clusters. 
Author Contributions: Conceptualization, L.Y.; methodology, L.Y.; software, L.Y.; validation, L.Y.; formal analysis, L.Y.; investigation, L.Y.; resources, L.Y. and Z.H.; writing-original draft preparation, L.Y.; writing一review and editing, L.Y.; funding acquisition, L.Y. and Z.H. All authors have read and agreed to the published version of the manuscript.

Funding: This research was funded by National Natural Science Foundation of China, grant number No. 51977156.

Conflicts of Interest: The authors declare no conflict of interest.

\section{References}

1. Tonkoski, R.; Tamrakar, U.; Meegahapola, L. Microgrid Stability Definitions, Analysis, and Examples. IEEE Trans. Power Syst. 2019, 8950, 1-17.

2. Parhizi, S.; Lotfi, H.; Khodaei, A. State of the Art in Research on Microgrids: A Review. IEEE Access 2015, 3 , 890-925. [CrossRef]

3. Goyal, M.; Ghosh, A. Microgrids interconnection to support mutually during any contingency. Sustain. Energy Grids Netw. 2016, 6, 100-108. [CrossRef]

4. Fini, M.H.; Golshan, M.E.H. Determining optimal virtual inertia and frequency control parameters to preserve the frequency stability in islanded microgrids with high penetration of renewables. Electr. Power Syst. Res. 2018, 154, 13-22. [CrossRef]

5. Soni, N.; Doolla, S.; Chandorkar, M.C. Analysis of Frequency Transients in Isolated Microgrids. IEEE Trans. Ind. Appl. 2017, 53, 5940-5951. [CrossRef]

6. Zhao, Z.; Yang, P.; Wang, Y. Dynamic Characteristics Analysis and Stabilization of PV-Based Multiple Microgrid Clusters. IEEE Trans. Smart Grid. 2019, 10, 805-818. [CrossRef]

7. Bullich-massagué, E.; Díaz-gonzález, F.; Aragüés-peñalba, M. Microgrid clustering architectures. Appl. Energy 2018, 212, 340-361. [CrossRef]

8. Bevrani, H.; Ise, T.; Miura, Y. Virtual synchronous generators: A survey and new perspectives. Int. J. Electr. Power Energy Syst. 2014, 54, 244-254. [CrossRef]

9. Van, T.V. Virtual synchronous generator: An element of future grids. In Proceedings of the 2010 IEEE PES Innovative Smart Grid Technologies Conference Europe (ISGT Europe), Gothenberg, Sweden, 11-13 October 2010; pp. 1-7.

10. Liu, J.; Miura, Y.; Ise, T. Comparison of Dynamic Characteristics between Virtual Synchronous Generator and Droop Control in Inverter-Based Distributed Generators. IEEE Trans. Power Electron. 2016, 31, 3600-3611. [CrossRef]

11. Serban, I.; Ion, C.P. Microgrid control based on a grid-forming inverter operating as virtual synchronous generator with enhanced dynamic response capability. Int. J. Electr. Power Energy Syst. 2017, 89, 94-105. [CrossRef]

12. Liu, J.; Yang, D.; Yao, W. PV-based virtual synchronous generator with variable inertia to enhance power system transient stability utilizing the energy storage system. Prot. Control Mod. Power Syst. 2017, 2, 39. [CrossRef]

13. Wang, Y.; Wang, C.; Xu, L. Adjustable Inertial Response From the Converter With Adaptive Droop Control in DC Grids. IEEE Trans. Smart Grid 2019, 10, 3198-3209. [CrossRef]

14. Pappu, V.A.K.; Chowdhury, B.; Bhatt, R. Implementing frequency regulation capability in a solar photovoltaic power plant. In Proceedings of the North American Power Symposium 2010, Arlington, TX, USA, 26-28 September 2010; pp. 1-6.

15. Zarina, P.P.; Mishra, S.; Sekhar, P.C. Exploring frequency control capability of a PV system in a hybrid PV-rotating machine-without storage system. Int. J. Electr. Power Energy Syst. 2014, 60, 258-267. [CrossRef]

16. Farrokhabadi, M.; König, S.; Cañizares, C.A. Battery Energy Storage System Models for Microgrid Stability Analysis and Dynamic Simulation. IEEE Trans. Power Syst. 2018, 33, 2301-2312. [CrossRef]

17. Arani, A.A.K.; Gharehpetian, G.B.; Abedi, M. Review on Energy Storage Systems Control Methods in Microgrids. Int. J. Electr. Power Energy Syst. 2019, 107, 745-757. [CrossRef]

18. Assi, Z. Control of a population of battery energy storage systems for frequency response. Int. J. Electr. Power Energy Syst. 2020, 115, 105463.

19. Lopes, J.A.P.; Moreira, C.L.; Madureira, A.G. Defining control strategies for MicroGrids islanded operation. IEEE Trans. Power Syst. 2006, 21, 916-924. [CrossRef] 
20. Wu, D.; Tang, F.; Dragicevic, T. Autonomous Active Power Control for Islanded AC Microgrids with Photovoltaic Generation and Energy Storage System. IEEE Trans. Energy Convers. 2014, 29, 882-892. [CrossRef]

21. Pogaku, N.; Prodanovic, M.; Green, T.C. Modeling, Analysis and Testing of Autonomous Operation of an Inverter-Based Microgrid. IEEE Trans. Power Electron. 2007, 22, 613-625. [CrossRef]

22. Majumder, R.; Chaudhuri, B.; Ghosh, A. Improvement of Stability and Load Sharing in an Autonomous Microgrid Using Supplementary Droop Control Loop. IEEE Trans. Power Syst. 2010, 25, 796-808. [CrossRef]

23. Barklund, E.; Pogaku, N.; Prodanovi, M. Energy Management in Autonomous Microgrid Using Stability-Constrained Droop Control of Inverters. IEEE Trans. Power Electron. 2008, 23, 2346-2352. [CrossRef]

24. Yang, L.; Hu, Z.; Xie, S. Adjustable virtual inertia control of supercapacitors in PV-based AC microgrid cluster. Electr. Power Syst. Res. 2019, 173, 71-85. [CrossRef]

25. Guanghu, X.; Chen, C.; Qu, S. The influence of induction motor inertia constant on small-signal stability. Electr. Power Syst. Res. 2005, 74, 197-202. [CrossRef]

26. Hui, L.; Shengquan, L.; Haiting, J. Damping control strategies of inter-area low-frequency oscillation for DFIG-based wind farms integrated into a power system. Int. J. Electr. Power Energy Syst. 2014, 61, $279-287$.

(C) 2020 by the authors. Licensee MDPI, Basel, Switzerland. This article is an open access article distributed under the terms and conditions of the Creative Commons Attribution (CC BY) license (http://creativecommons.org/licenses/by/4.0/). 